\title{
Keratin-Based Epidermal Green Autofluorescence is a Common Biomarker of
}

\section{Organ Injury}

Mingchao Zhang ${ }^{1,2}$, Yujia Li ${ }^{1,2}$, Jiucun Wang ${ }^{3,4}$, Huiru Tang ${ }^{3,4,5}$, Zhong Yang ${ }^{4,6}$,

Danhong $\mathrm{Wu}^{7}$, Hao $\mathrm{He}^{1}$, Yue Tao ${ }^{1,2}$, Zhaoxia Yang ${ }^{1,2}$, Dhruba Tara Maharjan ${ }^{1}$, Sijia Wang ${ }^{8}$, Xingdong Chen ${ }^{3,4,9}$, Li Jin ${ }^{3,4}$, Weihai Ying ${ }^{1,2, \#}$

${ }^{1}$ Med-X Research Institute and School of Biomedical Engineering, Shanghai Jiao Tong

University, Shanghai, China; ${ }^{2}$ Collaborative Innovation Center for Genetics and Development,

Shanghai, China; ${ }^{3}$ State Key Laboratory of Genetic Engineering, Collaborative Innovation

Center for Genetics and Development, School of Life Sciences, Fudan University, Shanghai,

China, ${ }^{4}$ Human Phenome Institute, Fudan University, Shanghai, China, ${ }^{5}$ Metabonomics and

Systems Biology Laboratory at Shanghai International Centre for Molecular Phenomics, Fudan

University, Shanghai, China; ${ }^{6}$ School of Life Sciences, Fudan University, Shanghai, China;

${ }^{7}$ Department of Neurology, Shanghai Fifth People's Hospital, Fudan University, Shanghai,

China; ${ }^{8}$ CAS Key Laboratory of Computational Biology, CAS-MPG Partner Institute for

Computational Biology, Shanghai Institute of Nutrition and Health, Chinese Academy of

Sciences, Shanghai, China; ${ }^{9}$ Fudan University Taizhou Institute of Health Sciences, Taizhou, Jiangsu Province, China. 
\#: Corresponding author:

Weihai Ying, Ph.D.

Professor, School of Biomedical Engineering and Med-X Research Institute

Shanghai Jiao Tong University

1954 Huashan Road

Shanghai, 200030, P.R. China

E-mail: weihaiy@sjtu.edu.cn

Running head: Epidermal Autofluorescence is a Biomarker 


\begin{abstract}
It is critical to discover biomarkers for non-invasive evaluation of inflammation and oxidative stress in human body - two key common pathological factors in numerous diseases. Our study has indicated keratin 1 (K1)-based epidermal autofluorescence (AF) as a novel biomarker of this type: Epidermal green AF intensity was significantly associated with both lipopolysaccharide (LPS) dosages and the serum levels of multiple cytokines in LPS-administered mice; and the green AF intensity of mouse's skin or skin cell cultures was highly associated with the dosages of oxidative stress inducers. The epidermal AF had the characteristic polyhydral structure of the stratum spinosum. The polyhydral structure with highly increased green AF was also found in the Dorsal Index Finger's skin of $45.59 \%$ of acute ischemic stroke (AIS) patients, which was profoundly decreased in the stroke patients' skin during recovery phase. Our study has further obtained multiple lines of evidence indicating K1 as an origin of the pathological insult-induced AF, including the finding showing that $\mathrm{K} 1 \mathrm{siRNA}$ administration significantly attenuated the increase in the mice' epidermal AF. Collectively, our study has indicated that K1-based epidermal AF is the first biomarker for non-invasive evaluation of inflammation and oxidative stress in the body. This finding forms a basis for novel keratin's AF-based biomedical imaging technology for not only screening and diagnosis of inflammation- and oxidative stress-associated diseases including AIS and COVID-19, but also evaluations of the inflammation and oxidative stress in the body of natural populations.
\end{abstract}

Keywords: Biomarker; Inflammation; Oxidative stress; Autofluorescence; Keratin. 


\section{Introduction}

Inflammation and oxidative stress are two key common pathological factors of multiple major diseases such as acute ischemic stroke (AIS) ${ }^{1-4}$. Chronic systemic inflammation and oxidative stress are closely related, which can also promote development of a number of diseases ${ }^{5-7}$. However, there has been no pracatical method or biomarker that can be used for determining non-invasively the levels of inflammation and oxidative stress in human body. Therefore, it is of critical significance to search for both the methods and the biomarkers of this type.

Human autofluorescence (AF) from the advanced glycation endproduct (AGE)-modified collagen and elastin of dermis has shown promise for non-invasive diagnosis of diabetes and diabetes-related pathology ${ }^{8}$. Epidermal AF may also become a novel endogenous reporter of certain pathological insults, if the pathological insults can induce changes of epidermal AF. Keratins, melanin, NADH and FAD are four known epidermal fluorophores ${ }^{9,10}$. There are increased inflammation and reactive oxygen species (ROS) in impaired organs and other parts of the body such as the blood circulation in a number of diseases ${ }^{11-16}$. Therefore, we hypothesized that inflammation and ROS in the body may lead to changes of epidermal AF by affecting certain epidermal fluorophores, which may become an endogenous reporter of the levels of inflammation and ROS in the body.

Keratins play multiple biological roles in epithelium, including intermediate filament formation ${ }^{17}$ and inflammatory responses ${ }^{18}$. Keratins have also been used as a tumor biomarker ${ }^{19}$. Keratin 1 (K1) / keratin 10 (K10) heterodimer is a hallmarker for keratinocyte differentiation ${ }^{20}$, which is localized selectively in suprabasal differentiated keratinocytes ${ }^{21}$. 
However, there has been little information regarding the biomedical value of keratin's AF.

In our current study, we tested our hypothesis that epidermal AF may be a biomarker for the levels of inflammation and oxidative stress in the body. Our study has indicated that K1-based epidermal AF is a biomarker of this type, which forms a basis for novel, keratin-based biomedical imaging technology for non-invasive evaluation of the inflammation and oxidative stress in human body.

\section{Materials and Methods}

\section{Materials}

All chemicals were purchased from Sigma (St. Louis, MO, USA) except where noted. Male C57BL/6SLACBL/6Slac mice, ICR mice, and BALB/cASlac-nu nude mice of SPF grade were purchased from SLRC Laboratory (Shanghai, China).

\section{Studies on Human Subjects}

The stroke study was conducted according to a protocol approved by the Ethics Committee of Shanghai Fifth People's Hospital Affiliated to Fudan University. The human subjects in the study included healthy controls and AIS patients, with average age of $63.91 \pm$ 0.95 and $63.96 \pm 1.13$ years of old, respectively. Three months after they were released from hospital, nine of the AIS patients with average age of $63.89 \pm 4.22$ years of old were also studied as the stroke patients in recovery phase. The UVC-exposure study on the human subjects with average age of $24.58 \pm 0.71$ was conducted according to a protocol approved by the Ethics Committee for Human Subject Studies of Shanghai Ninth Hospital affiliated to 
Shanghai Jiao Tong University School of Medicine.

\section{Exposures of UV radiation}

After C57BL/6SLACBL/6Slac mice, ICR mice, or BALB/cASlac-nu nude mice at the weight between 18 - $35 \mathrm{~g}$ were anesthetized briefly with chloral hydrate at the dosage of $1 \mathrm{ml}$ $3.5 \%(\mathrm{w} / \mathrm{v}) / 100 \mathrm{~g}$, the ears of the mice were exposed to a UVC lamp (TUV 25W /G25 T8, Philips, Hamburg, Germany) with the power density of $0.55 \pm 0.05 \mathrm{~mW} / \mathrm{cm}^{2}$. For the study on human subjects, the index fingers of human subjects were exposed to a radiation dosage of 2.4 $\pm 0.1 \mathrm{~J} / \mathrm{cm}^{2}$ from the UVC lamp at the power density of $2.0 \pm 0.1 \mathrm{~mW} / \mathrm{cm}^{2}$.

\section{Imaging of the AF of mouse's skin}

The AF of mouse's ears was imaged under a two-photon fluorescence microscope (A1 plus, Nikon Instech Co., Ltd., Tokyo, Japan), with the excitation wavelength of $488 \mathrm{~nm}$ and the emission wavelength of $500-530 \mathrm{~nm}$. The AF was quantified by the following approach: Sixteen spots with the size of approximately $100 \mathrm{X} 100 \mu \mathrm{m}^{2}$ on the scanned images were selected randomly. After the average AF intensities of each layer were calculated, the sum of the average AF intensities of all layers of each spot was calculated, which is defined as the AF intensity of each spot. If the value of average AF intensity of certain layer is below 45, the AF signal of the layer was deemed background noise, which was not counted into the sum. The AF spectra of the mice were determined by using a two-photon fluorescence microscope (A1 plus,

Nikon Instech Co., Ltd., Tokyo, Japan). After the imaging, the images were analyzed automatically. 


\section{Determinations of the AF and the AF spectrum of human subjects'skin}

A portable AF imaging equipment was used to determine the skin' AF of the human subjects. The excitation wavelength was $485 \mathrm{~nm}$, and the emission wavelength was $500-550$ $\mathrm{nm}$. By using a portable equipment for measuring AF spectrum, we determined the spectra of the skin' AF of the human subjects. The emitting light of the wavelength longer than $450 \mathrm{~nm}$ was detected, when the excitation wavelength of $445 \mathrm{~nm}$ was used.

\section{Western blot assays}

The lysates of the skin were centrifuged at $12,000 \mathrm{~g}$ for $20 \mathrm{~min}$ at $4^{\circ} \mathrm{C}$. The protein concentrations of the samples were quantified using BCA Protein Assay Kit (Pierce Biotechonology, Rockford, IL, USA). As described previously ${ }^{22}$, a total of $50 \mu \mathrm{g}$ of total protein was electrophoresed through a 10\% SDS-polyacrylamide gel, which were then electrotransferred to $0.45 \mu \mathrm{m}$ nitrocellulose membranes (Millipore, CA, USA). The blots were incubated with a monoclonal Anti-Cytokeratin 1 (ab185628, Abcam, Cambridge, UK) (1:4000 dilution) or actin (1:1000, sc-58673, Santa Cruz Biotechnology, Inc., Dallas, TX, USA ) with $0.05 \%$ BSA overnight at $4^{\circ} \mathrm{C}$, then incubated with HRP conjugated Goat Anti-Rabbit IgG $(\mathrm{H}+\mathrm{L})$ (1:4000, Jackson ImmunoResearch, PA, USA) or HRP conjugated Goat Anti-mouse IgG (1:2000, HA1006, HuaBio, Zhejiang Province, China). An ECL detection system (Thermo Scientific, Pierce, IL, USA) was used to detect the protein signals. The intensities of the bands were quantified by densitometry using Image $\mathrm{J}$.

\section{Immunohistochemistry}


Ten $\mu \mathrm{m}$ paraffin-sections of skin were obtained by a Leica Cryostat, mounted onto poly-L-lysine coated slides and stored at room temperature. After the sections were incubated in Xylene three times, the skin sections were sequentially dehydrated in $100 \% \mathrm{EtOH}, 95 \% \mathrm{EtOH}$ and $70 \%$ EtOH. After two washes with PBS, the sections were blocked in $10 \%$ goat serum for $1 \mathrm{hr}$, which were incubated in monoclonal Anti-Cytokeratin 1 (ab185628, abcam, Cambridge, UK) (1:1000 dilution), containing $1 \%$ goat serum at $4{ }^{\circ} \mathrm{C}$ overnight. After three washes in PBS, the sections were incubated with Alexa Fluor 647 goat anti-rabbit $\lg$ (1:1000 dilution) (Invitrogen, CA,USA) for $1 \mathrm{hr}$ in darkness at RT, followed by staining in $0.2 \%$ DAPI solution (Beyotime, Haimen, Jiangsu Province, China) for $5 \mathrm{~min}$. The sections were mounted in Fluorescence Mounting Medium (Beyotime, Haimen, Jiangsu Province, China). To compare the intensity of fluorescence in each sample, at least three randomly picked fields in each section were photographed under a Leica microscope.

\section{Histology}

Skin biopsies from the ears of the mice were obtained, which were placed immediately in 4\% (w/v) paraformaldehyde buffer. After $12-24$ hrs, paraffin embedding procedure was conducted on the samples. Hematoxylin / Eosin staining was performed according to the manufacturer's protocol (Beyotime, Haimen, Jiangsu Province, China).

\section{Laser-based delivery of keratin 1 siRNA into mouse's skin}

Male C57BL/6SLACBL/6SlacMice were briefly anesthetized with 3.5\% (w/v) chloral hydrate $(1 \mathrm{ml} / 100 \mathrm{~g})$. After exposure to laser, the ears of the mouse were transfected with $\mathrm{K} 1$ 
siRNA using Lipofectamine 3000 following the manufacturer's instructions (Thermo Fisher

Scientific, Waltham, MA, USA). The sequences of the mouse K1 siRNA were CUCCCAUUUGGUUUGUAGCTT and UGACUGGUCACUCUUCAGCTT (GenePharma, Shanghai, China).

\section{Statistical analyses}

All data are presented as mean \pm SEM. Data were assessed by one-way ANOVA, followed by Student - Newman - Keuls post hoc test, except where noted. $P$ values less than 0.05 were considered statistically significant.

\section{Results}

\section{Epidermal green AF intensity was significantly associated with both LPS dosages and} serum levels of multiple cytokines in LPS-administered C57BL/6SLAC mice

We determined the effects of LPS, an inducer of inflammation, on the epidermal green AF of C57BL/6SLAC mice's ears: LPS produced dose-dependent increases in the epidermal green AF intensity at both 3 and 7 days after the LPS administration (Figs. 1A and 1B), with the AF intensity being significantly associated with the LPS dosages (Fig. 1C). The spatial distribution of the LPS-induced AF is distinctly polyhedral, exhibiting the characteristic structure of the suprabasal keratinocytes at the stratum spinosum (Fig. 1A). Orthographic green AF images of the mouse's skin showed that the LPS-induced AF increases occurred only at certain layer of the skin with the thickness of approximately $10 \mu \mathrm{m}$, which was approximately $10-20 \mu \mathrm{m}$ apart from the outer surface of the epidermis (Fig. 1D). H\&E staining of the skin showed that the 
thickness of the epidermis was approximately $25-30 \mu \mathrm{m}$, while the thickness of the stratum corneum was less that $3 \mu \mathrm{m}$ (Supplemental Fig. 1A), indicating that the origin of the AF was localized between the stratum corneum and the dermis.

Three days after the LPS administration, the epidermal green AF intensity was found to be significantly associated with both LPS dosages and the Log-transformed serum levels of multiple cytokines of the mice, including IL-1 $\beta$, IL-2, IL-5, IL-6, IL-10, IL-12(p40), monocyte chemoattractant protein-1 (MCP-1) and G-CSF (Table 1). The epidermal green AF intensity was also significantly associated with both LPS dosages and the serum levels of MIP-1 $\alpha$, MIP-1 $\beta$ and RANTES/CCL5 (Table 2). In contrast, neither the LPS dosage nor the epidermal AF intensity was significantly associated with either the Log-transformed serum levels (Supplemental Table 1) or the serum levels of multiple cytokines (Supplemental Table 2).

2. Green AF intensity of mouse's skin or skin cell cultures was highly associated with the dosages of oxidative stress inducers

We determined the effects of oxidative stress on the green AF of B16-F10 cells, a skin cell line: $\mathrm{H}_{2} \mathrm{O}_{2}$ produced concentration-dependent increases in the green AF intensity (Figs. 2A and Fig. 2B), with the AF intensity being highly associated with the $\mathrm{H}_{2} \mathrm{O}_{2}$ concentrations at both 1 and 6 hrs after the $\mathrm{H}_{2} \mathrm{O}_{2}$ exposures (Figs. 2C). Since UVC can induce increased ROS leading to cell death and tissue damage ${ }^{23,24}$, we also applied UVC as an oxidative stress inducer: UVC irradiation produced dose-dependent increases in the green AF intensity of the cells (Figs. 2D and 2E), with the AF intensity being highly associated with the UVC dosages at both 1 and 6 hrs after the UVC irradiation (Fig. 2F). 
UVC produced dose-dependent increases in the green AF intensity of the ear's skin of C57BL/6SLAC mice, assessed at 1, 6, or 24 hrs after the UVC exposures (Figs. 2G and 2H), with its dosages being highly associated with the AF intensity (Fig. 2I). UVC also produced significant increases in the epidermal green AF intensity of both nude mice and ICR mice (Figs. 2J and 2K). The spectra of both basal and UVC-induced AF of C57BL/6SLAC mice, ICR mice and nude mice were similar, reaching maximal AF intensity at 470 - $500 \mathrm{~nm}$ when the excitation wavelength was $800 \mathrm{~nm}$ under a two-photon fluorescence microscope (Fig. 2L). Orthographic green AF images of the UVC-irradiated C57BL/6SLAC mouse's skin were highly similar with those of the LPS-induced AF, with its spatial distribution being characteristically polyhedral (Fig. 2M). UVC also induced a significant increase in the green AF of human subjects' skin (Supplemental Figs. 2A, 2B and 2C).

\section{Green AF intensity was significantly increased in AIS patients'skin}

There are significant increases in oxidative stress and inflammatory responses in both the CNS and the other parts of the body of AIS patients ${ }^{25-27}$. To determine if our findings from the animal and cell culture studies are also applicable to major oxidative stress- and inflammation-associated diseases, we investigated if there is also increased green AF in AIS patients' skin: The green AF intensity of both right and left Dorsal Index Fingers' skin of the AIS patients was remarkably higher than that of the healthy controls (Fig. 3A). In the skin of at least one of their Dorsal Index Fingers, there was characteristic polyhedral structures with highly or mildly increased green AF in 31 and 15 patients out of the 68 AIS patients, respectively (Fig. 3B). The characteristic polyhedral structures were highly similar with those observed in the 
LPS- or UVC-exposed ears' skin of mice. In either one or both of their Dorsal Index Finger's skin of $45.59 \%$ of AIS patients and $1.72 \%$ healthy controls, there were polyhedral structures with highly increased green AF (Fig. 3C). In either one or both of their Dorsal Index Finger's skin of $22.06 \%$ of AIS patients and $13.79 \%$ healthy controls, there were only polyhedral structures with mildly increased green AF (Fig. 3C). Both the average AF intensity of the polyhedral structures with highly increased green AF and the average AF intensity of the Dorsal Index Finger's skin without the the polyhedral structures of the AIS patients was markedly higher than that of the healthy controls (Fig. 3D). ROC analysis using the AF intensity of either the right or the left Dorsal Index Fingers' skin as sole parameter showed that the AUC for differentiating healthy controls and AIS patients reached 0.8271 and 0.8261 , respectively (Figs. 3E and 3F). The spectrum of the AF of the AIS patient's skin was virtually identical as that of the healthy controls (Fig. 3G).

Three months after nine of the AIS patients were released from the hospital, the patients' scores of National Institutes of Health Stroke Scale (NIHSS) - a widely used scale for rating stroke severity ${ }^{28}$ - were significantly reduced compared with those in their acute phase (Figs. 3H). The AF intensity of the released patients was also profoundly decreased in both their right and left Dorsal Index Fingers'skin, compared with that of the patients in their acute phase (Figs.

3I and 3J). Six of the nine released patients had decreased AF intensity at both Dorsal Index Fingers' skin, while two of the nine released patients had decreased AF intensity at one of their Dorsal Index Fingers'skin (Fig. 3K).

\section{4. $\mathrm{K} 1$ is an origin of the UVC-induced increase in the epidermal AF of mice}


We investigated the origins of the oxidative stress- and inflammation-induced increases in the epidermal AF, suggesting that K1 and K10 may be the origin of the epidermal AF: 1) The AF is selectively localized at the stratum spinosum (Fig. 1A and Fig. 2G, Fig. 1D, Fig. 2M), where the sole autofluorescent molecules that are selectively localized are K1 and K10 ${ }^{21}$. 2) The spectrum of the UVC-induced green AF (Fig. 2L) matches that of keratins' AF ${ }^{29}$. 3) The structure and localization of the LPS- (Fig. 1A) and UVC-induced AF (Figs. 2G and 2J) match the structure and localization of $\mathrm{K} 1 / \mathrm{K} 10$ heterodimers in the epidermis, which form unique, highly dense bundles that are parallel with the cell membranes of the polyhedral suprabasal cells 30,31. Immunostaining assays of the ear's skin of C57BL/6SLAC mice using anti-K1 antibody also showed that the polyhedral structure of the K1-positive regions (Supplemental Fig. 3) matched the polyhedral structure of the LPS- (Fig. 1A) and UVC-induced AF (Figs. 2G and 2J). Our study has further excluded the possibility that the other three molecules among the four known epidermal autofluorescent molecules ${ }^{10}$ may be the origin of the AF: First, none of melanin, FAD and NADH is localized selectively at the stratum spinosum; second, the excitation wavelength for NAD(P)H' AF is in UVA range ${ }^{32}$, which is far below the excitation wavelength used in our study; third, when it significantly increased the epidermal AF intensity of C57BL/6SLAC mouse's ears at $1 \mathrm{hr}$ after the UVC exposures (Figs. 2G and 2H), UVC significantly decreased the FAD level of the skin (Supplemental Figure 4); and fourth, UVC induced a significant increase in the AF of the ear's skin of ICR mice (Figs. 2J and 2K), a strain of albino mice that are melanin-deficient ${ }^{33}$.

Since K1 is associated with K10 as K1/K10 heterodimers in differentiated keratinocytes 21,34,35, we determined if K1 and K10 exist as monomers or heterodimers in the ear's skin of 
C57BL/6SLAC mice. Western blot assays on K1 using normal denaturing conditions showed that in the samples of the ear's skin, there was a distinct band at approximately $127 \mathrm{kDa}$, while the level of K1 monomers at the $67 \mathrm{kDa}$ band was exceedingly low (Supplemental Figs. 5A). When the samples were prepared in $8 \mathrm{M}$ urea, Western blot assays on K1 showed that the 127 $\mathrm{kDa}$ band disappeared with appearance of both a $67 \mathrm{kDa}$ and a $59 \mathrm{kDa}$ band, while Western blot assays on K10 showed an increased band at $60 \mathrm{kDa}$ (Supplemental Figs. 5B). These observations have indicated that the $127 \mathrm{kDa}$ band is the band of the $\mathrm{K} 1 / \mathrm{K} 10$ heterodimers. We further determined the effects of LPS and UVC exposures on the levels of K1 and K10: LPS induced significant decreases in both $\mathrm{K} 1$ and K10 levels of the ears one day after the mice were i.p. administered with 0.5 or $1 \mathrm{mg} / \mathrm{kg}$ LPS (Figs. 4A, 4B and 4C). In contrast, UVC produced a K1 decrease but a K10 increase of C57BL/6SLAC mice's ears one day after the mice were exposed to UVC (Figs. 4D, 4E and 4F). Since K1 decrease, but not K10 change, was correlated with both LPS- and UVC-induced AF increases, the K1 decrease may be causative to the AF increases. Both $\mathrm{H}_{2} \mathrm{O}_{2}$ and UVC also induced significant $\mathrm{K} 1$ decreases of B16-F10 cells (Supplemental Figs. 6A, 6B, 6C and 6D).

We determined if the antioxidant N-acetyl cysteine (NAC) can block the UVC-induced $\mathrm{K} 1$ decrease and the AF increase. NAC administration dose-dependently attenuated both the UVC-induced K1 decrease (Figs. 4G and 4H) and the AF increase (Figs. 4I and 4J). This finding has indicated that prevention of the UVC-induced $\mathrm{K} 1$ decrease is associated with prevention of the UVC-induced AF increase, which is also consistent with our proposal that the $\mathrm{K} 1$ decrease plays a significant role in the AF increase. To further identify the role of $\mathrm{K} 1$ in the UVC-induced AF increase, we applied laser-based technology to deliver K1 siRNA into the 
skin of the mouse's ears, showing a marked increase in the Cy5-labeled siRNA inside the skin (Supplemental Fig. 7). Both Western blot (Figs. 4K and 4L) and immunostaining assays (Fig. 4M) showed that the laser-based K1 siRNA administration led to a K1 decrease. The K1 siRNA administration also significantly attenuated the UVC-induced AF increase (Figs. 4N and 40).

\section{Discussion}

This study has provided strong evidence indicating the epidermal green AF as a novel endogenous reporter of the inflammation and oxidative stress in the body: Our animal studies and skin cell culture studies have found significant associations between the green AF intensity and the dosages of both the inflammation inducer and the oxidative stress inducers. Our study on AIS - a major oxidative stress- and inflammation-associated disease, has also indicated that the skin's green AF intensity is a novel indicator of the disease: The AIS patients had a remarkable increase in their skin's AF intensity compared with the healthy controls, which was significantly decreased during their recovery phase. Moreover, the increased skin's AF of the AIS patients had highly similar polyhedral structures as those found in the skin of LPS- and UVC-administered mice. These findings have collectively suggested that the AF is a common biomarker of the pathological insults in the body of both human and the animals.

Our study has not only provided first information indicating profound biomedical value of keratin's AF, but also discovered a novel property of keratins' AF: In addition to its basal AF, $\mathrm{K} 1$ 's AF is inducible by inflammation and UVC. Moreover, our study has indicated that the green AF is a biomarker of 'real damage' of cells and tissues, since the intensity of LPS-, UVC-, or $\mathrm{H}_{2} \mathrm{O}_{2}$-induced green $\mathrm{AF}$ is significantly associated with the dosages of these pathological 
insults that can dose-dependently produce cellular and tissue damage. In contrast, current approach for evaluating the risk of developing such major diseases as AIS can provide only methematical probability of developing the diseases based on the risk factors a person has ${ }^{36}$.

Our study has indicated $\mathrm{K} 1$ as an origin of the oxidative stress- and inflammation-induced epidermal AF increases: First, the K1 siRNA-produced K1 decrease significantly attenuated the UVC-induced AF increase; second, when it attenuated UVC-produced K1 decrease, NAC significantly attenuated the UVC-induced AF increase; third, the increased $\mathrm{AF}$ is selectively localized at the stratum spinosum, where the sole known autofluorescent molecules that are selectively localized are $\mathrm{K} 1$ and $\mathrm{K} 10^{21}$; fourth, the spectrum of the UVC-induced green AF matches that of keratins' $\mathrm{AF}^{29}$, and fifth, the structure and localization of the LPS- and UVC-induced AF match those of $\mathrm{K} 1 / \mathrm{K} 10$ heterodimers in the epidermis ${ }^{30,31}$. Moreover, our studies have excluded the possibility that the other three known epidermal autofluorescent molecules ${ }^{10}$ are the origin of the AF increases. It has been reported that cleavage of collagen by various proteases can lead to increased $\mathrm{AF}^{37}$. We proposed that LPS and UVC may induce the increased green AF by the following mechanisms: LPS and UVC induce cleavage of full-length $\mathrm{K} 1$, leading to exposures of the K1's domains that generate the AF. Our observation that NAC blocks both the UVC-induced K1 decrease and the UVC-induced AF increase is consistent with this proposal. Future studies are warranted to further investigate the mechanisms underlying the AF increases.

Our study has provided first evidence indicated profound and extensive applications of the keratin's AF for non-invasive diagnosis and community screening of inflammation- and oxidative stress-associated diseases, as indicated by our study on the skin's AF of the AIS 
patients: By using the AF intensity of either right or left Dorsal Index Fingers'skin as the sole parameter, AUC in ROC analysis for differentiating the healthy controls and the AIS patients reached 0.82 . With applications of $\mathrm{AI}$ and additions of the information regarding both the structural properties of the AF images and clinical symptoms, the sensitivity and specificity of this novel diagnostic method may be markedly enhanced. Increased inflammation and/or oxidative stress have also been found in the patients' body of numerous diseases including lung cancer ${ }^{13,14}$, Parkinson's disease ${ }^{15,16}$ and COVID-19 ${ }^{38,39}$. Therefore, our AF-based approach may also be used for evaluating the pathological state of the patients of these diseases, which has been supported by our preliminary studies showing that each of the diseases studied has its characteristic changes of the pattern of their skin's green $A F^{40,41}$. Our current study has also indicated that the keratin's AF-based approach may become the first non-invasive method for monitoring the levels of multiple cytokines in the body, which further indicates the great potential of this AF imaging technology for non-invasive evaluations of patients' key biological parameters. Compared with major medical imaging technology including MRI, CT and PET technology, our AF-based imaging technology are remarkably more economic, time-efficient and non-invasive.

Collectively, our findings have formed a basis for establishing a novel, keratin's AF-based biomedical imaging technology. This technology has multiple distinct merits for non-invasive screening and diagnosis of inflammation- and oxidative stress-associated diseases. Moreover, this technology may also be used for non-invasive, economic and rapid evaluations of the levels of inflammation and oxidative stress in the body of natural populations, which are critically needed in health management. 
bioRxiv preprint doi: https://doi.org/10.1101/564112; this version posted May 30, 2020. The copyright holder for this preprint (which was not certified by peer review) is the author/funder. All rights reserved. No reuse allowed without permission.

\section{Acknowledgment:}

The authors would like to acknowledge the financial support by two research grants

from a Major Special Program Grant of Shanghai Municipality (Grant \# 2017SHZDZX01) (to

W.Y. and L.J.) and a Major Research Grant from the Scientific Committee of Shanghai

Municipality \#16JC1400500 (to L.J.) and \#16JC1400502 (to W.Y.). 


\section{References:}

1 Taniguchi, K. \& Karin, M. NF-kappaB, inflammation, immunity and cancer: coming of age. Nat Rev Immunol 18, 309-324, doi:10.1038/nri.2017.142 (2018).

2 Lin, M. T. \& Beal, M. F. Mitochondrial dysfunction and oxidative stress in neurodegenerative diseases. Nature 443, 787-795, doi:10.1038/nature05292 (2006).

3 Xia, W., Han, J., Huang, G. \& Ying, W. Inflammation in ischaemic brain injury: current advances and future perspectives. Clin Exp Pharmacol Physiol 37, 253-258, doi:10.1111/j.1440-1681.2009.05279.x (2010).

4 Ying, W. \& Xiong, Z. G. Oxidative stress and NAD ${ }^{+}$in ischemic brain injury: current advances and future perspectives. Curr Med Chem 17, 2152-2158 (2010).

5 Furman, D. et al. Chronic inflammation in the etiology of disease across the life span. Nat Med 25, 1822-1832, doi:10.1038/s41591-019-0675-0 (2019).

6 Reuter, S., Gupta, S. C., Chaturvedi, M. M. \& Aggarwal, B. B. Oxidative stress, inflammation, and cancer: how are they linked? Free Radic Biol Med 49, 1603-1616, doi:10.1016/j.freeradbiomed.2010.09.006 (2010).

7 Steven, S. et al. Vascular Inflammation and Oxidative Stress: Major Triggers for Cardiovascular Disease. Oxid Med Cell Longev 2019, 7092151, doi:10.1155/2019/7092151 (2019).

8 Moran, C. et al. Type 2 diabetes, skin autofluorescence, and brain atrophy. Diabetes 64, 279-283, doi:10.2337/db14-0506 (2015).

9 Pena, A., Strupler, M., Boulesteix, T. \& Schanne-Klein, M. Spectroscopic analysis of keratin endogenous signal for skin multiphoton microscopy. Opt Express 13, 
6268-6274 (2005).

10 Bader, A. N. et al. Fast nonlinear spectral microscopy of in vivo human skin. Biomed Opt Express 2, 365-373, doi:10.1364/BOE.2.000365 (2011).

11 Jayaraj, R. L., Azimullah, S., Beiram, R., Jalal, F. Y. \& Rosenberg, G. A. Neuroinflammation: friend and foe for ischemic stroke. J Neuroinflammation 16, 142, doi:10.1186/s12974-019-1516-2 (2019).

12 Rodrigo, R. et al. Oxidative stress and pathophysiology of ischemic stroke: novel therapeutic opportunities. CNS Neurol Disord Drug Targets 12, 698-714, doi:10.2174/1871527311312050015 (2013).

13 Karin, M. \& Greten, F. R. NF-kappaB: linking inflammation and immunity to cancer development and progression. Nat Rev Immunol 5, 749-759, doi:10.1038/nri1703 (2005).

14 Peddireddy, V., Siva Prasad, B., Gundimeda, S. D., Penagaluru, P. R. \& Mundluru, H. P. Assessment of 8-oxo-7, 8-dihydro-2'-deoxyguanosine and malondialdehyde levels as oxidative stress markers and antioxidant status in non-small cell lung cancer. Biomarkers 17, 261-268, doi:10.3109/1354750X.2012.664169 (2012).

15 Kaur, K., Gill, J. S., Bansal, P. K. \& Deshmukh, R. Neuroinflammation - A major cause for striatal dopaminergic degeneration in Parkinson's disease. J Neurol Sci 381, 308-314, doi:10.1016/j.jns.2017.08.3251 (2017).

16 Trist, B. G., Hare, D. J. \& Double, K. L. Oxidative stress in the aging substantia nigra and the etiology of Parkinson's disease. Aging Cell 18, e13031, doi:10.1111/acel.13031 (2019). 
17 Jacob, J. T., Coulombe, P. A., Kwan, R. \& Omary, M. B. Types I and II Keratin Intermediate Filaments. Cold Spring Harb Perspect Biol 10, doi:10.1101/cshperspect.a018275 (2018).

18 Roth, W. et al. Keratin 1 maintains skin integrity and participates in an inflammatory network in skin through interleukin-18. J Cell Sci 125, 5269-5279, doi:10.1242/jcs.116574 (2012).

19 Karantza, V. Keratins in health and cancer: more than mere epithelial cell markers. Oncogene 30, 127-138, doi:10.1038/onc.2010.456 (2011).

20 Woodcock-Mitchell, J., Eichner, R., Nelson, W. G. \& Sun, T. T. Immunolocalization of keratin polypeptides in human epidermis using monoclonal antibodies. J Cell Biol 95, 580-588 (1982).

21 Sun, T. T. et al. Keratin classes: molecular markers for different types of epithelial differentiation. J Invest Dermatol 81, 109s-115s (1983).

22 Sheng, C. et al. $\mathrm{NAD}(+)$ administration significantly attenuates synchrotron radiation X-ray-induced DNA damage and structural alterations of rodent testes. Int J Physiol Pathophysiol Pharmacol 4, 1-9 (2012).

23 Zhang, X., Rosenstein, B. S., Wang, Y., Lebwohl, M. \& Wei, H. Identification of possible reactive oxygen species involved in ultraviolet radiation-induced oxidative DNA damage. Free Radic Biol Med 23, 980-985, doi:10.1016/s0891-5849(97)00126-3 (1997).

24 Guyton, K. Z., Xu, Q. \& Holbrook, N. J. Induction of the mammalian stress response gene GADD153 by oxidative stress: role of AP-1 element. Biochem J 314 ( Pt 2), 
547-554, doi:10.1042/bj3140547 (1996).

25 Seet, R. C. et al. Oxidative damage in ischemic stroke revealed using multiple biomarkers. Stroke 42, 2326-2329, doi:10.1161/STROKEAHA.111.618835 (2011).

26 Ciancarelli, I., Di Massimo, C., De Amicis, D., Carolei, A. \& Tozzi Ciancarelli, M. G. Evidence of redox unbalance in post-acute ischemic stroke patients. Curr Neurovasc $\operatorname{Res}$ 9, 85-90 (2012).

27 Dominguez, C. et al. Oxidative stress after thrombolysis-induced reperfusion in human stroke. Stroke 41, 653-660, doi:10.1161/STROKEAHA.109.571935 (2010).

28 Lyden, P. Using the National Institutes of Health Stroke Scale: A Cautionary Tale. Stroke 48, 513-519, doi:10.1161/STROKEAHA.116.015434 (2017).

29 Zvyagin, A. V. et al. Imaging of zinc oxide nanoparticle penetration in human skin in vitro and in vivo. J Biomed Opt 13, 064031, doi:10.1117/1.3041492 (2008).

30 Reichelt, J., Bussow, H., Grund, C. \& Magin, T. M. Formation of a normal epidermis supported by increased stability of keratins 5 and 14 in keratin 10 null mice. Mol Biol Cell 12, 1557-1568, doi:10.1091/mbc.12.6.1557 (2001).

31 P A Coulombe, K. M. B., and C-H Lee. Keratins and the Skin. Cell Architecture and Function., 665-671. (2013.).

32 Patterson, G. H., Knobel, S. M., Arkhammar, P., Thastrup, O. \& Piston, D. W. Separation of the glucose-stimulated cytoplasmic and mitochondrial NAD $(\mathrm{P}) \mathrm{H}$ responses in pancreatic islet beta cells. Proc Natl Acad Sci U S A 97, 5203-5207, doi:10.1073/pnas.090098797 (2000).

33 Beermann, F. et al. Rescue of the albino phenotype by introduction of a functional 
tyrosinase gene into mice. EMBO J 9, 2819-2826 (1990).

34 Moll, R., Franke, W. W., Schiller, D. L., Geiger, B. \& Krepler, R. The catalog of human cytokeratins: patterns of expression in normal epithelia, tumors and cultured cells. Cell 31, 11-24 (1982).

35 Eichner, R., Bonitz, P. \& Sun, T. T. Classification of epidermal keratins according to their immunoreactivity, isoelectric point, and mode of expression. J Cell Biol 98, 1388-1396 (1984).

36 O'Donnell, M. J. et al. Global and regional effects of potentially modifiable risk factors associated with acute stroke in 32 countries (INTERSTROKE): a case-control study. Lancet 388, 761-775, doi:10.1016/S0140-6736(16)30506-2 (2016).

37 Deyl, Z., Praus, R., Sulcova, H. \& Goldman, J. N. Fluorescence of collagen properties of tyrosine residues and another fluorescent element in calf skin collagen. FEBS Lett 5, 187-191, doi:10.1016/0014-5793(69)80328-5 (1969).

38 Huang, C. et al. Clinical features of patients infected with 2019 novel coronavirus in Wuhan, China. Lancet 395, 497-506, doi:10.1016/S0140-6736(20)30183-5 (2020).

39 Mehta, P. et al. COVID-19: consider cytokine storm syndromes and immunosuppression. Lancet 395, 1033-1034, doi:10.1016/S0140-6736(20)30628-0 (2020).

40 Zhang, M. et al. Selectively increased autofluorescence at certain locations of skin may become a novel diagnostic biomarker for lung cancer. bioRxiv, 315440., doi: https://doi.org/10.1101/315440 (2020).

$41 \mathrm{Wu}, \mathrm{D}$. , et al. Selectively increased autofluorescence at fingernails and certain 
bioRxiv preprint doi: https://doi.org/10.1101/564112; this version posted May 30, 2020. The copyright holder for this preprint (which was not certified by peer review) is the author/funder. All rights reserved. No reuse allowed without permission.

regions of skin: A potential novel diagnostic biomarker for Parkinson disease. bioRxiv 322222, doi:https://doi.org/10.1101/322222 (2018). 


\section{Figure Legends:}

Figure 1. Epidermal green AF intensity was significantly associated with LPS dosages in LPS-administered C57BL/6SLAC mice. (A) LPS produced increased epidermal green AF intensity of the skin of the mice' ears at both 3 and 7 days after intraperitoneal injection of 0.5 or $1 \mathrm{mg} / \mathrm{kg}$ LPS. (B) LPS produced significant and dose-dependent increases in the epidermal green $\mathrm{AF}$ intensity at both 3 and 7 days after the LPS administration. $\mathrm{N}=19-28 .{ }^{*}, P<$ 0.05 ; **, $P<0.01$; ***, $P<0.001$. (C) The AF intensity was significantly associated with the LPS dosages. The data were obtained from one representative experiment out of the three independent experiments. $\mathrm{N}=5$ - 6. (D) Orthographic green AF images of the mouse's skin showed that the LPS-induced AF increases occurred only at certain layer of the skin with the thickness of approximately $10 \mu \mathrm{m}$. The images of XY axis (100 $\mu \mathrm{m}$ X $100 \mu \mathrm{m}), \mathrm{YZ}$ axis $(100$ $\mu \mathrm{m} X 100 \mu \mathrm{m})$ and $\mathrm{XZ}$ axis $(100 \mu \mathrm{m} X 100 \mu \mathrm{m})$ were shown. $\mathrm{N}=6$.

Fig. 2. Green AF intensity of C57BL/6SLAC mouse's skin or skin cell cultures was highly associated with the dosages of oxidative stress inducers. (A) $\mathrm{H}_{2} \mathrm{O}_{2}$ produced increased green AF intensity of B16-F10 cells at both 1 and 6 hrs after the $\mathrm{H}_{2} \mathrm{O}_{2}$ exposures. (B) $\mathrm{H}_{2} \mathrm{O}_{2}$ produced significant and concentration-dependent increases in the green AF intensity of B16-F10 cells at both 1 and 6 hrs after the $\mathrm{H}_{2} \mathrm{O}_{2}$ exposures. $\mathrm{N}=9 . *, P<0.05$; ***, $P<0.001$. The data were collected from three independent experiments. (C) The AF intensity was significantly associated with the $\mathrm{H}_{2} \mathrm{O}_{2}$ concentrations at both 1 and 6 hrs after the $\mathrm{H}_{2} \mathrm{O}_{2}$ exposures. The data were obtained from one representative experiment out of the three independent experiments. $\mathrm{N}=3$. (D) UVC irradiation produced increased green AF intensity 
of B16-F10 cells at both 1 and 6 hrs after the UVC irradiation. (E) UVC irradiation produced significant and dose-dependent increases in the green AF intensity of B16-F10 cells at both 1 and 6 hrs after the UVC irradiation. $\mathrm{N}=12 . * *, P<0.05 ; * * *, P<0.001$. The data were collected from three independent experiments. (F) The AF intensity was significantly associated with the UVC dosages at both 1 and $6 \mathrm{hrs}$ after the UVC irradiation. The data were obtained from one representative experiment out of the three independent experiments. $N=4$. (G) UVC irradiation produced increased green AF intensity of the ear's skin of C57BL/6SLAC mice at 1, 6, or 24 hrs after the UVC exposures. Scale bar $=20 \mu \mathrm{m} . \quad(\mathrm{H})$ UVC irradiation produced significant and dose-dependent increases in the green AF intensity of the ear's skin of C57BL/6SLAC mice at 1, 6, or 24 hrs after the UVC exposures. $\mathrm{N}=10-13 . *, P<0.05$; ***, $P<0.001$. (I) The AF intensity was significantly associated with the UVC dosages at 1,6 , or 24 hrs after the UVC exposures. The data were obtained from one representative experiment out of the three independent experiments. $\mathrm{N}=5-7$. (J) UVC produced increased epidermal green AF intensity of both nude mice and ICR mice at 3 - 6 hrs after the UVC exposures. Scale bar $=20$ $\mu \mathrm{m}$. (K) UVC produced significant increases in the epidermal green AF intensity of both nude mice and ICR mice at 3 - 6 hrs after the UVC exposures. $\mathrm{N}=4-5 . * *, P<0.01$; ***, $P<0.001$. (L) The spectra of both basal and UVC-induced AF of C57BL/6SLAC mice, ICR mice and nude mice were similar, reaching maximal AF intensity at $470-500 \mathrm{~nm}$ when the excitation wavelength was $800 \mathrm{~nm}$ under a two-photon fluorescence microscope. $\mathrm{N}=3$. The data are the representative of the spectrum of the skin's AF of one mice for each strain. (M) Orthographic green AF images of the mouse's skin indicated that the UVC-induced AF increases occurred only at certain layer of the skin with the thickness of approximately $10 \mu \mathrm{m}$. The images of XY 
axis $(100 \mu \mathrm{m} * 100 \mu \mathrm{m}$ square $), \mathrm{YZ}$ axis $(100 \mu \mathrm{m}$ in length, left column $)$ and $\mathrm{XZ}$ axis $(100 \mu \mathrm{m}$ in length, bottom column) were shown. $\mathrm{N}=6$.

Fig. 3. Green AF intensity was significantly increased in AIS patients' skin. (A) The green AF intensity of both right and left Dorsal Index Fingers' skin of the AIS patients was significantly higher than that of the healthy controls. $\mathrm{N}=58-68$. ***, $P<0.001$. (B) In the skin of at least one of their Dorsal Index Fingers, there was characteristic polyhedral structures with highly or mildly increased green AF in 31 and 15 patients out of the 68 AIS patients, respectively. 'Highly increased AF' or 'mildly increased AF' was defined as 'the AF that has more than or less than three times of the AF intensity of the healthy controls'. The AF images of the polyhedral structures with highly or mildly increased green AF as well as the AF images without the polyhedral structures were representatives of the AF images of the AIF patients and the healthy controls. (C) In either one or both of their Dorsal Index Finger's skin of $45.59 \%$ of AIS patients and $1.72 \%$ healthy controls, there were polyhedral structures with highly increased green AF. In either one or both of their Dorsal Index Finger's skin of $22.06 \%$ of AIS patients and $13.79 \%$ healthy controls, there were polyhedral structures with mildly increased green AF. . $\mathrm{N}=58$ - 68. (D) The average AF intensity of the polyhedral structures with highly increased green AF as well as the average AF intensity of the Dorsal Index Finger's skin without the the polyhedral structures of the AIS patients was markedly higher than that of the healthy controls. The number of the persons who had the polyhedral structures with highly increased AF was 1 and 31 for the Healthy Group and the AIS group, respectively. The number of the persons who had only the polyhedral structures with mildly increased AF was 8 and 15 for the Healthy 
Group and the AIS group, respectively. The number of the persons who did not have the polyhedral structures was 49 and 22 for the Healthy Group and the AIS group, respectively. ROC analysis using the AF intensity of the right Dorsal Index Fingers' skin as sole parameter showed that the AUC for differentiating healthy controls and AIS patients reached 0.8271. $(\mathrm{F})$ ROC analysis using the AF intensity of the left Dorsal Index Fingers' skin as sole parameter showed that the AUC for differentiating healthy controls and AIS patients reached 0.8261. (G) The spectrum of the AF of the AIS patient's skin was similar with that of the healthy controls. The data were obtained from one representative experiment out of the three independent experiments. $\mathrm{N}=3$. (H) Three months after nine of the AIS patients were released from the hospital, the NIHSS scores of the patients were profoundly reduced compared with those in their acute phase. $\mathrm{N}=9 ; \#, P<0.05$ (paired student t-test). (I) The AF intensity of the released patients was significantly decreased in both their right and left Dorsal Index Fingers' skin, compared with that of the patients in their acute phase. $\mathrm{N}=9 ; \quad \#, P<0.05$ (paired student t-test). (J) Six of the nine released patients had decreased AF intensity at both Dorsal Index Fingers' skin, while two of the nine released patients had decreased AF intensity at one of their Dorsal Index Fingers'skin.

Fig. 4. K1 is an origin of the UVC-induced increase in the epidermal AF of C57BL/6SLAC mice. (A) LPS induced changes of both $\mathrm{K} 1$ and $\mathrm{K} 10$ levels of the ears one day after the mice were i.p. administered with 0.5 or $1 \mathrm{mg} / \mathrm{kg}$ LPS. (B) LPS induced a significant decrease in the K1 levels of the ears one day after the mice were i.p. administered with 0.5 or $1 \mathrm{mg} / \mathrm{kg}$ LPS. N $=6 .^{*}, P<0.05$. (C) LPS induced a significant decrease in the K10 levels of the ears one day 
after the mice were i.p. administered with 0.5 or $1 \mathrm{mg} / \mathrm{kg}$ LPS. $\mathrm{N}=6$. $^{* *}, P<0.01$. (D) UVC produced a K1 decrease but a K10 increase of C57BL/6SLAC mouse's ears one day after the mice were exposed to UVC. (E) UVC produced a significant K1 decrease of C57BL/6SLAC mice's ears one day after the mice were exposed to UVC. $\mathrm{N}=7 * P<0.05$. (F) UVC produced a significant K10 increase of C57BL/6SLAC mouse's ears one day after the mice were exposed to UVC. $\mathrm{N}=7$ \#, $P<0.05$; \#\#, $P<0.01$ (student t-test). (G,H) NAC administration significantly and dose-dependently attenuated the UVC-induced $\mathrm{K} 1$ decrease. $\mathrm{N}=8$; $\#, P<$ 0.05 (student t-test). (I,J) NAC administration significantly and dose-dependently attenuated and the AF increase. $\mathrm{N}=6$; $*, P<0.05 ; * *, P<0.01$. (K,L) Western blot showed that the laser-based $\mathrm{K} 1$ siRNA administration led to a significant $\mathrm{K} 1$ decrease. $\mathrm{N}=7$. ${ }^{*}, P<0.05$. (M) Immunostaining assays showed that the laser-based K1 siRNA administration led to a decreased $\mathrm{K} 1$ level. $\mathrm{N}=3-5$. $(\mathrm{N}, \mathrm{O})$ The $\mathrm{K} 1$ siRNA administration significantly attenuated the UVC-induced AF increase. $\mathrm{N}=6-7 . * *, P<0.01 ; * * *, P<0.001$.

\section{Legends of Tables:}

Table 1. Epidermal green AF intensity was significantly associated with both LPS dosages and Log-transformed serum levels of multiple cytokines in LPS-administered

C57BL/6SLAC mice. Epidermal green AF intensity was significantly associated with both LPS dosages and the Log-transformed serum levels of IL-1 $\beta$, IL-2, IL-5, IL-6, IL-10, IL-12(p40), monocyte chemoattractant protein-1 (MCP-1) and G-CSF of C57BL/6SLAC mice's. Three days after the mice were administered with 0.5 or $1 \mathrm{mg} / \mathrm{kg}$ LPS, the epidermal green AF intensity of the ear's skin of the mice were determined. The serum levels of multiple 
cytokines of the mice were also determined. $\quad \mathrm{N}=4-6$.

Table 2. Epidermal green AF intensity was significantly associated with both LPS dosages and serum levels of three cytokines in LPS-administered C57BL/6SLAC mice. The LPS dosages were significantly associated with both LPS dosages and serum levels of MIP-1 $\alpha$, MIP-1 $\beta$ and RANTES/CCL5 . Three days after the mice were administered with 0.5 or $1 \mathrm{mg} / \mathrm{kg}$ LPS, the epidermal green AF intensity of the ear's skin of the mice were determined. The serum levels of the cytokines of the mice were also determined. $\quad \mathrm{N}=4-6$.

\section{Legends of Supplemental Figures:}

Supplemental Fig. 1. H\&E staining of the skin showed that the thickness of the epidermis was approximately 25 - $30 \mu \mathrm{m}$, while the thickness of the stratum corneum was less that $3 \mu \mathrm{m}$.

Supplemental Fig. 2. (A,B) UVC induced a significant increase in the green AF of the skin of human subjects. $\mathrm{N}=12$. \#\#\#, $P<0.05$ (paired Student t-test).

Supplemental Fig. 3. Immunostaining assays of the ear's skin of C57BL/6SLAC mice using anti-K1 antibody also showed that the polyhedral structure of the K1-positive regions matched the polyhedral structure of the LPS- and UVC-induced AF. $\quad \mathrm{N}=3-5$.

Supplemental Figure 4. UVC significantly decreased the FAD level of the skin. $\mathrm{N}=21$. \#\#, $P<$ 0.01 (student t-test). 
Supplemental Figs. 5. (A) Western blot assays on K1 using normal denaturing conditions showed that in the samples of the ear's skin, there was a distinct band at approximately $127 \mathrm{kDa}$, while the level of $\mathrm{K} 1$ monomers at the $67 \mathrm{kDa}$ band was exceedingly low. When the samples were prepared in $8 \mathrm{M}$ urea, Western blot assays on $\mathrm{K} 1$ showed that the $127 \mathrm{kDa}$ band disappeared with appearance of both a $67 \mathrm{kDa}$ and a $59 \mathrm{kDa}$ band. $\mathrm{N}=3$. (B) When the samples were prepared in $8 \mathrm{M}$ urea, Western blot assays on K10 showed an increased band at $60 \mathrm{kDa}$. N $=3$.

Supplemental Figs. 6. (A,B) $\mathrm{H}_{2} \mathrm{O}_{2}$ induced a significant $\mathrm{K} 1$ decrease of B16-F10 cells. $\mathrm{N}=8$. *, $P<0.05$. The data were collected from two independent experiments. (C,D) UVC induced a significant $\mathrm{K} 1$ decrease of $\mathrm{B} 16-\mathrm{F} 10$ cells. $\mathrm{N}=9 . \#, P<0.05$ (student t-test). The data were collected from three independent experiments.

Supplemental Fig. 7. Laser-based technology was applied to deliver K1 siRNA into the skin of the mouse's ears, showing a marked increase in the Cy5-labeled siRNA inside the skin. $\mathrm{N}=3$.

\section{Legends of Supplemental Table:}

Supplemental Table 1. Neither the LPS dosage nor the epidermal AF intensity was significantly associated with the Log-transformed serum levels of multiple cytokines 3 days after LPS administration. Three days after the mice were administered with 0.5 or 1 $\mathrm{mg} / \mathrm{kg}$ LPS, the epidermal green AF intensity of the ear's skin of the mice was determined. The Log-transformed serum levels of multiple cytokines were also determined. $\mathrm{N}=4-6$. 
Supplemental Table 2. Neither the LPS dosage nor the epidermal AF intensity was significantly associated with the serum levels of multiple cytokines 3 days after LPS administration. Three days after the mice were administered with 0.5 or $1 \mathrm{mg} / \mathrm{kg}$ LPS, the epidermal green AF intensity of the ear's skin of the mice was determined. The serum levels of multiple cytokines were also determined. $\mathrm{N}=4-6$. 
a

b ${ }^{\text {con }}$

$0.5 \mathrm{mg} / \mathrm{kg}$

$1.0 \mathrm{mg} / \mathrm{kg}$

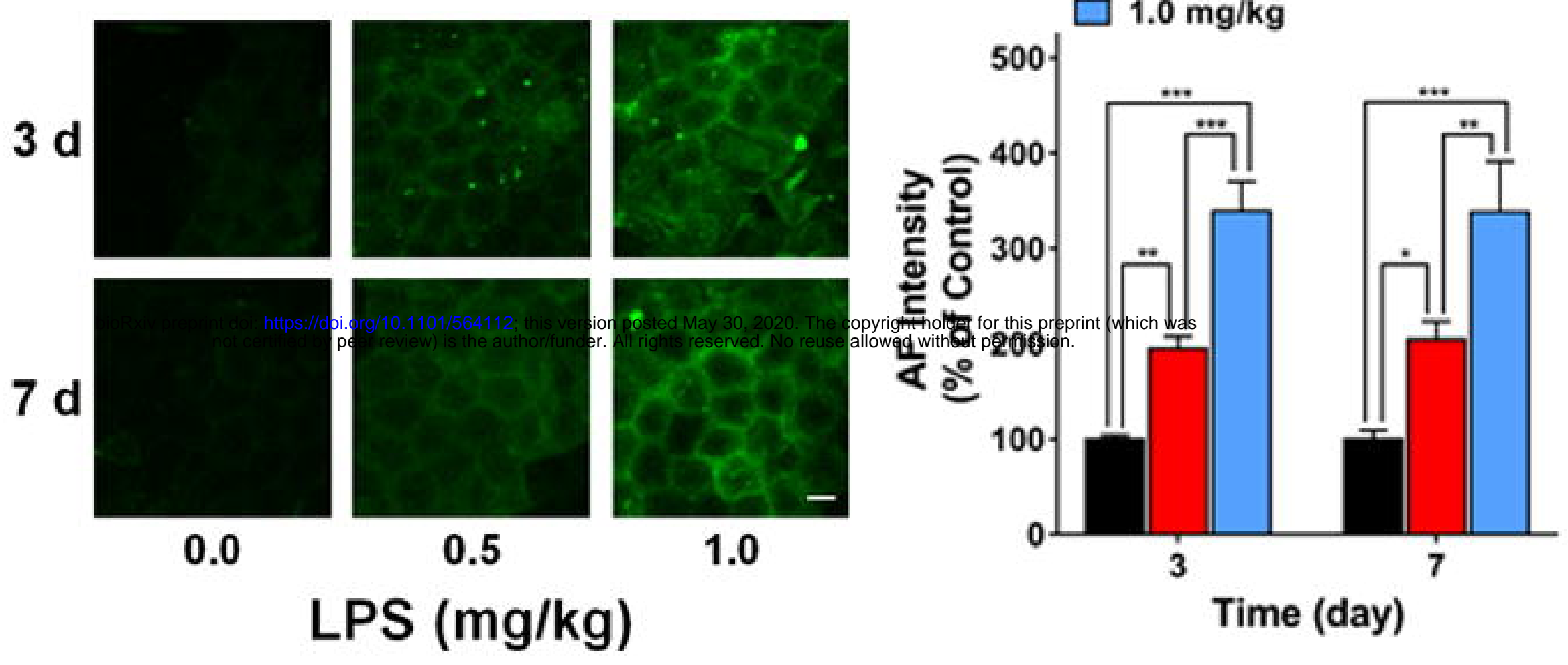

C

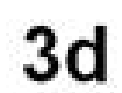

$7 d$

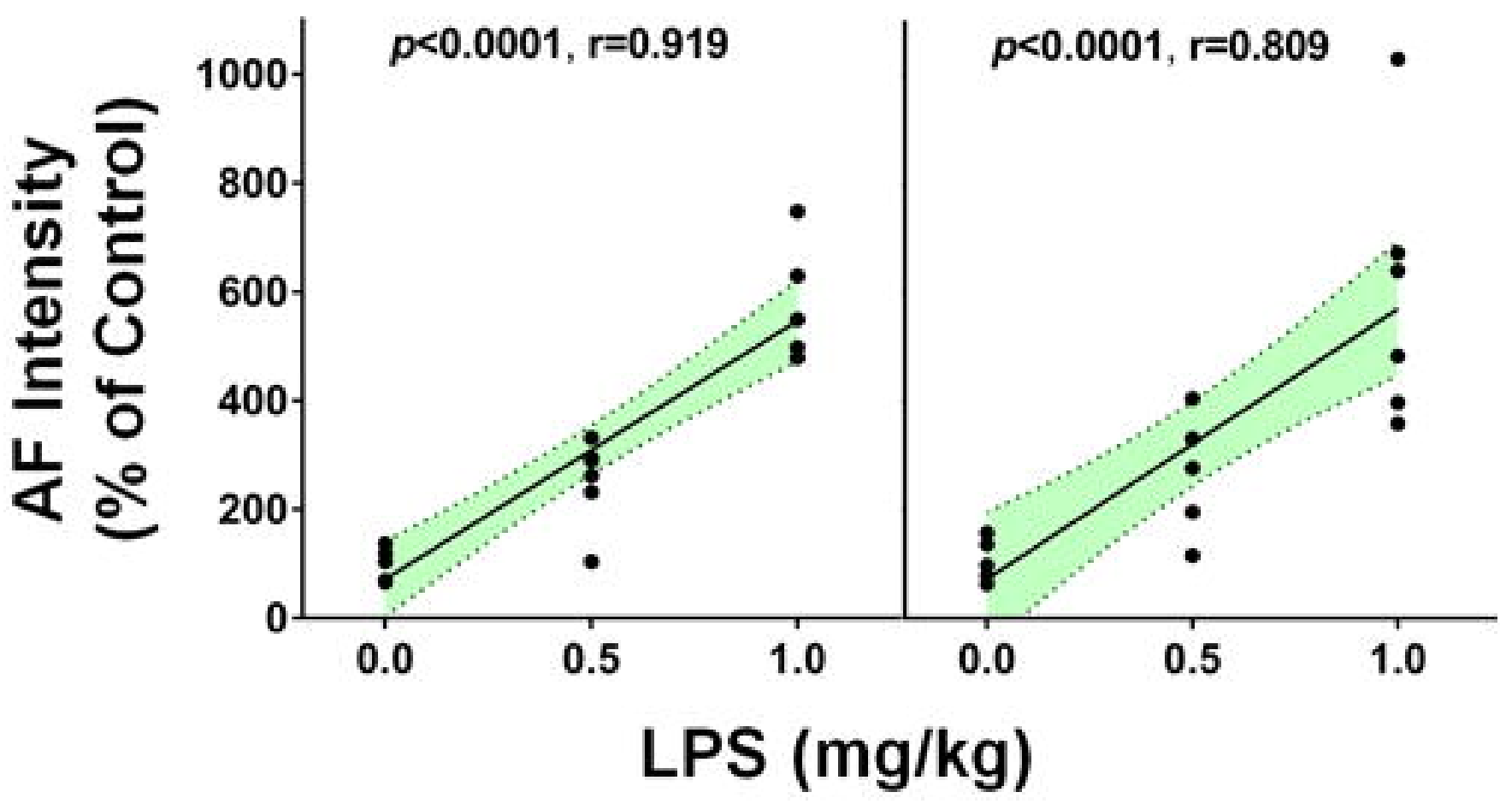

d

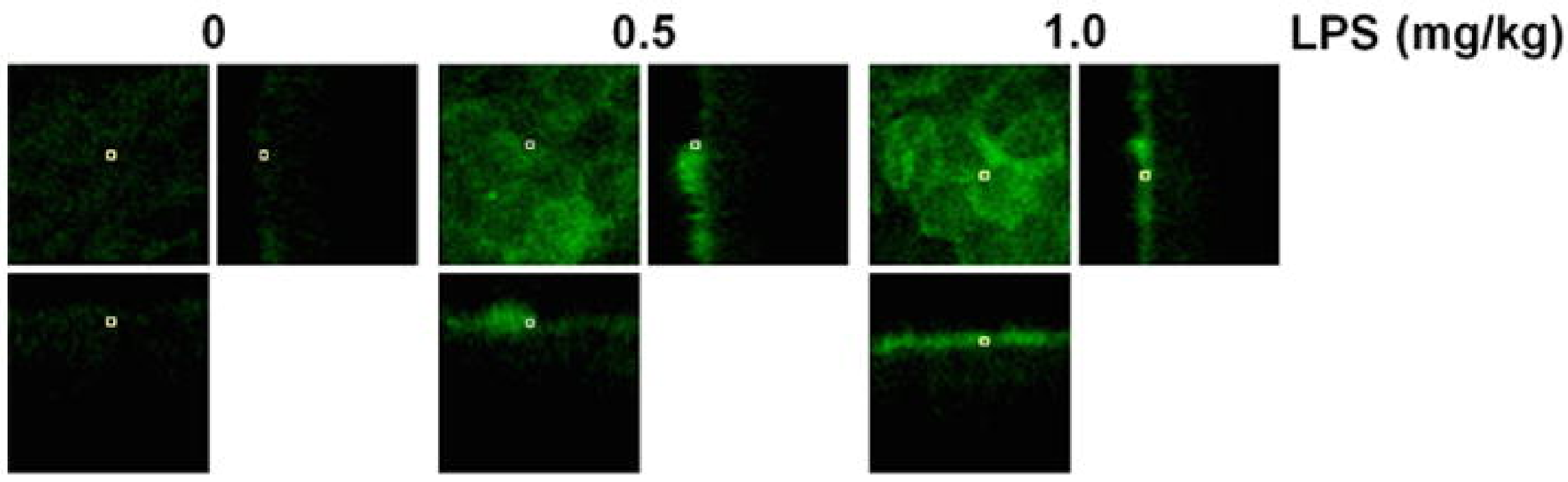


a

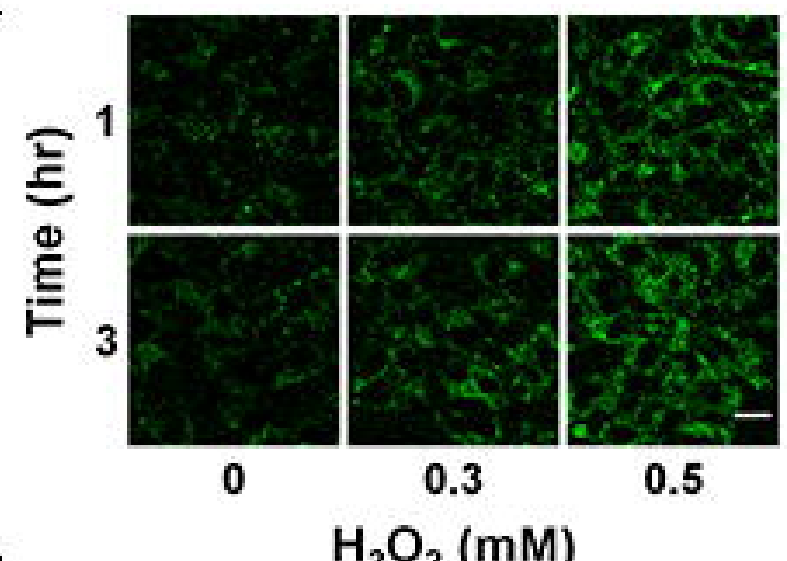

d

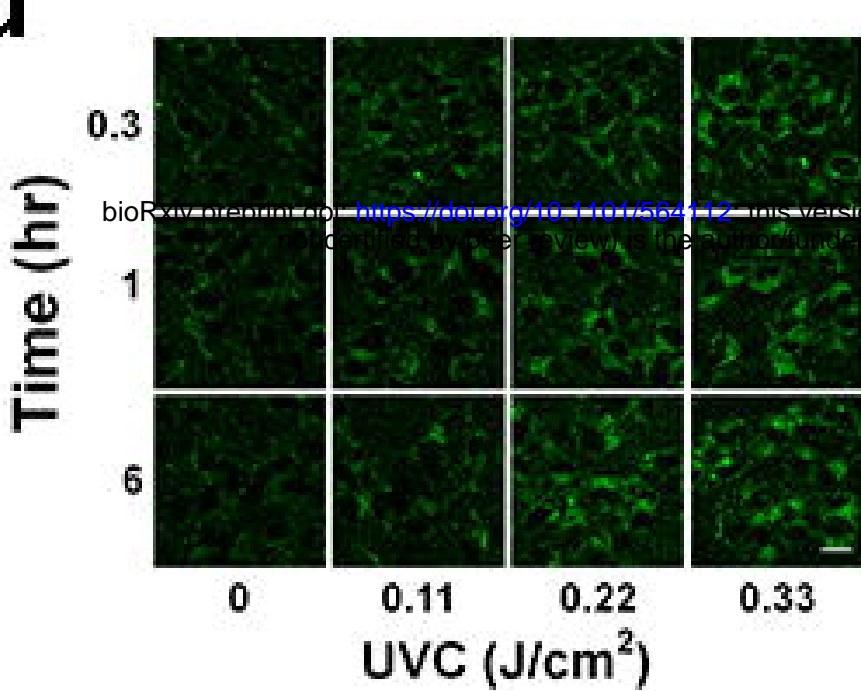

g

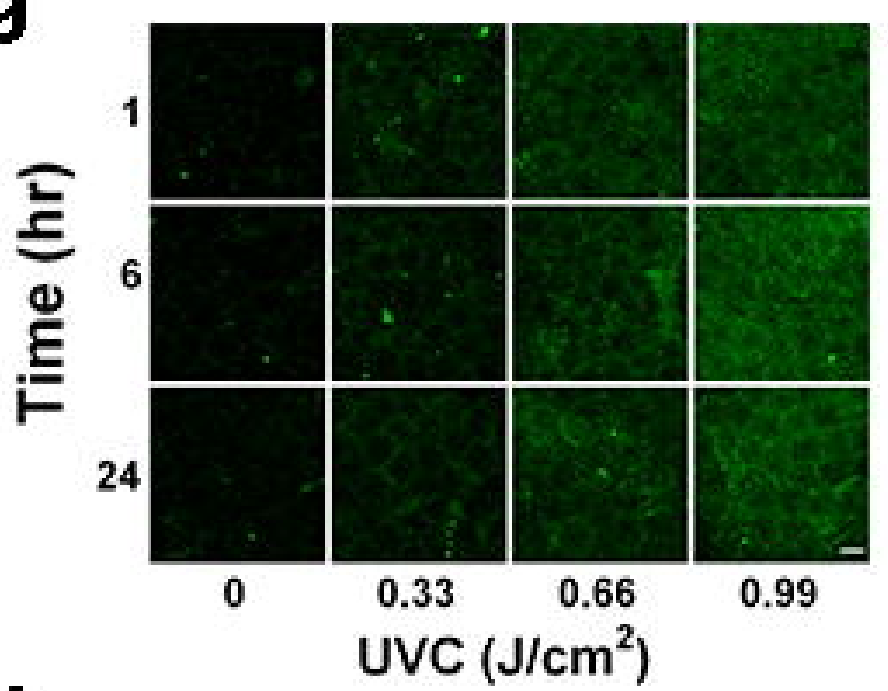

j

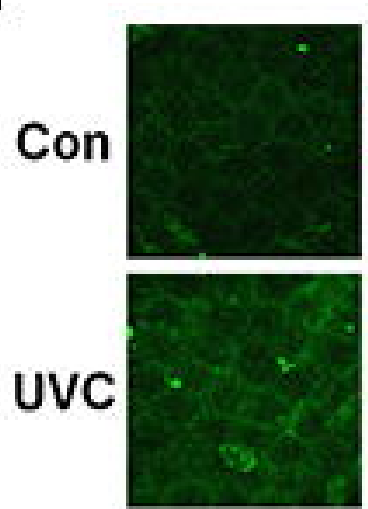

C57
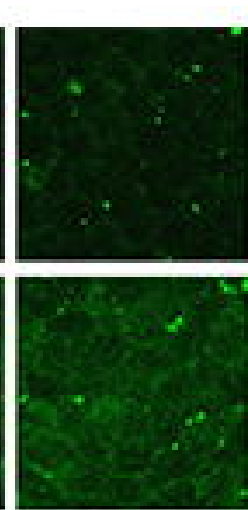

ICR

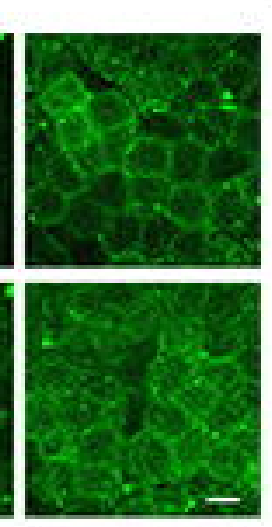

Nude a 它 $\begin{gathered}\mathrm{Con} \\ 0.5 \mathrm{mMM} \mathrm{H} \mathrm{H}_{2} \mathrm{O}_{2}\end{gathered}$

C

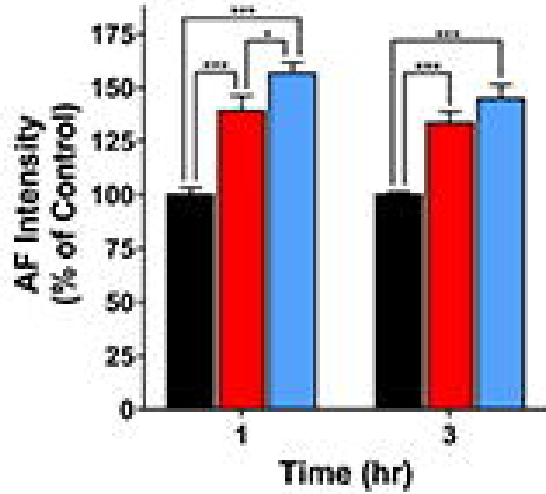

e

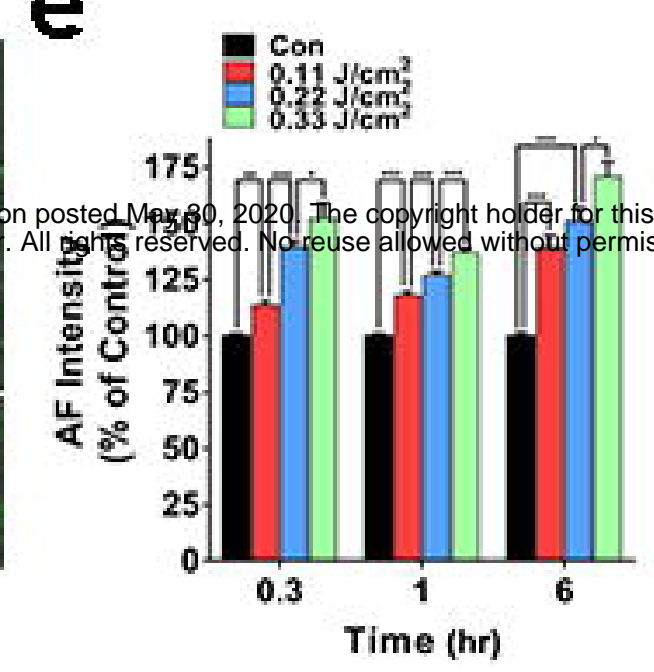

h

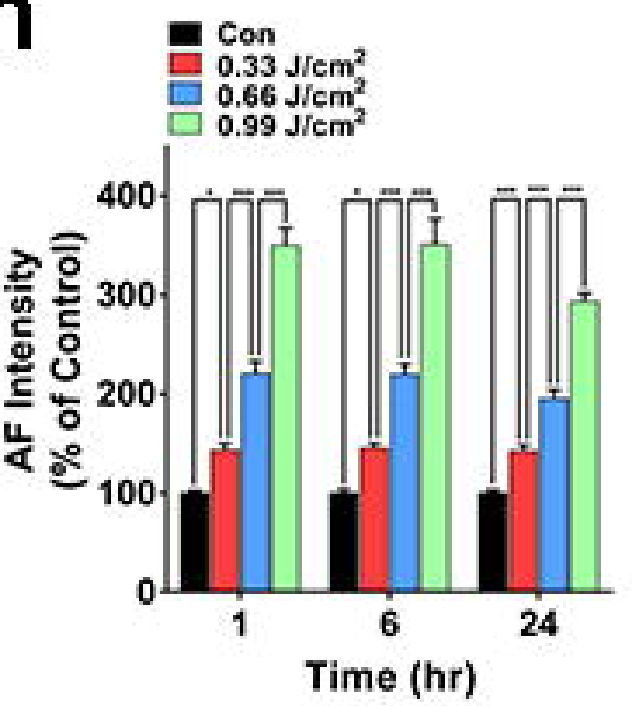

K E con

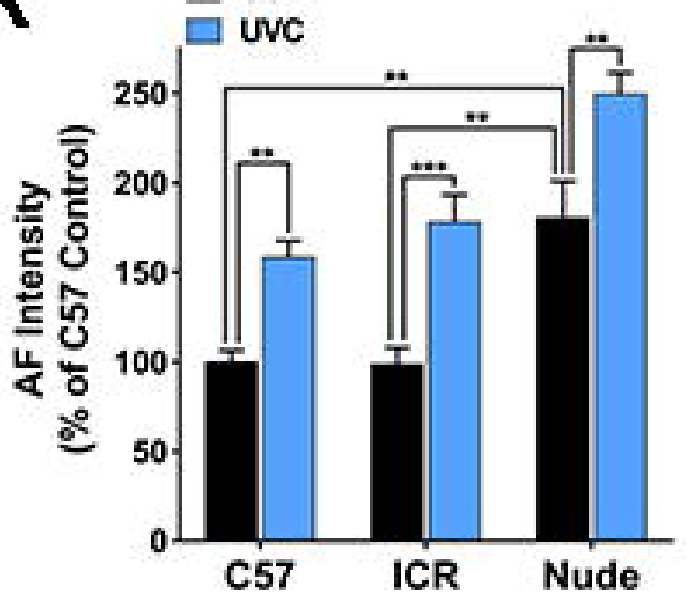

1

i
$1 \mathrm{hr}$

$3 \mathrm{hr}$

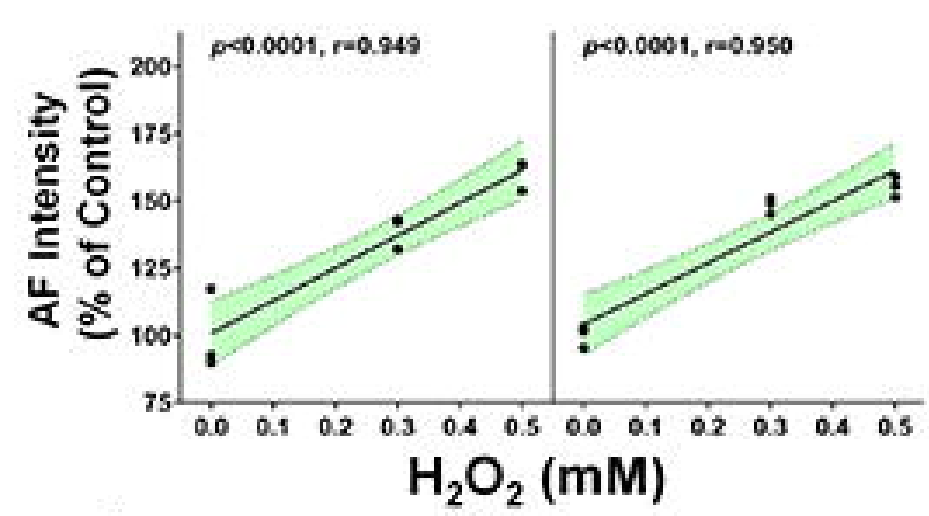

$0.3 \mathrm{hr}$

$1 \mathrm{hr}$

$6 \mathrm{hr}$
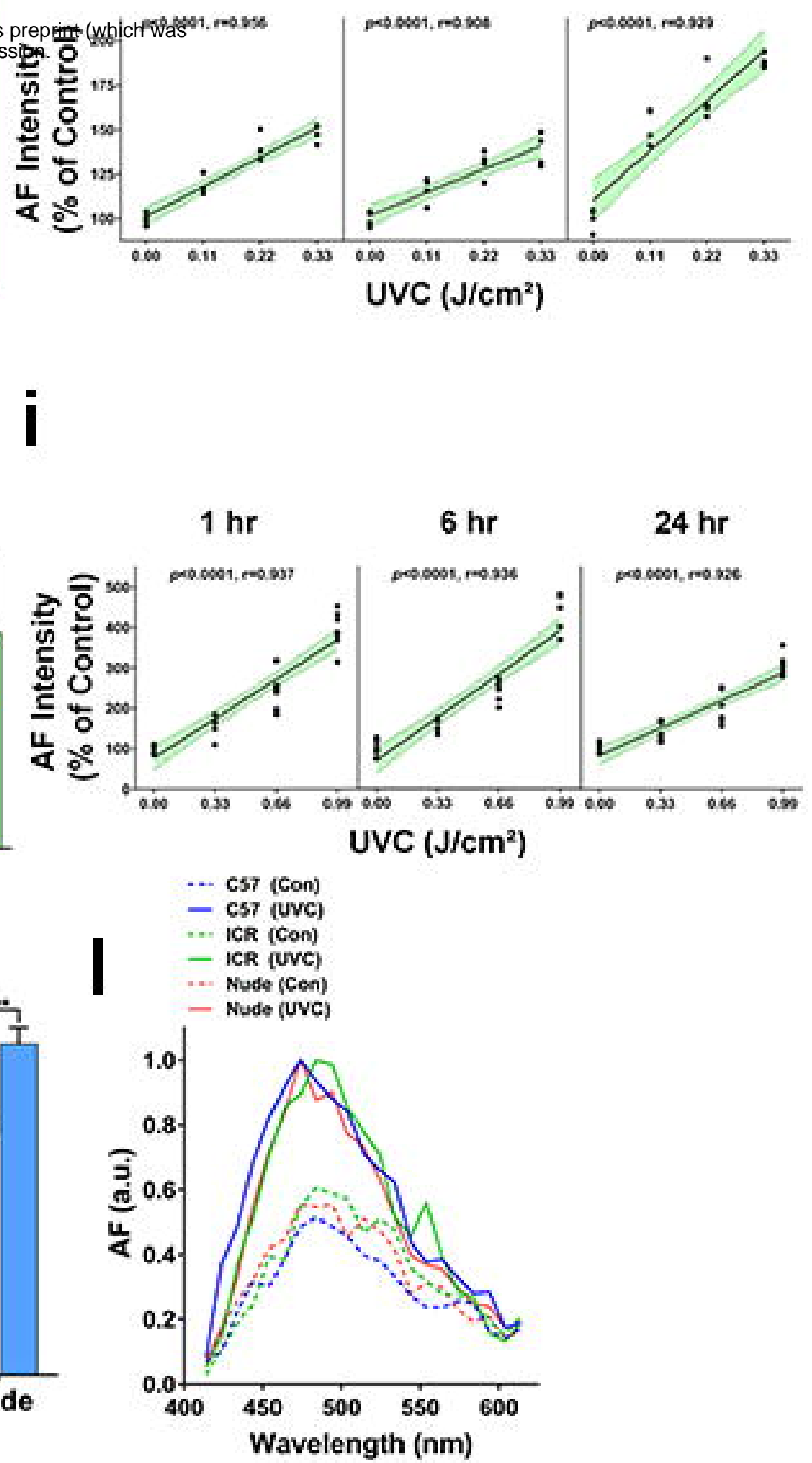

m

0
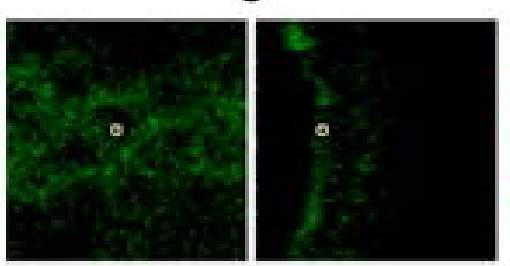

0.33

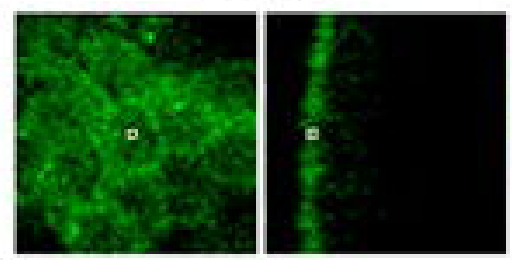

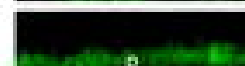
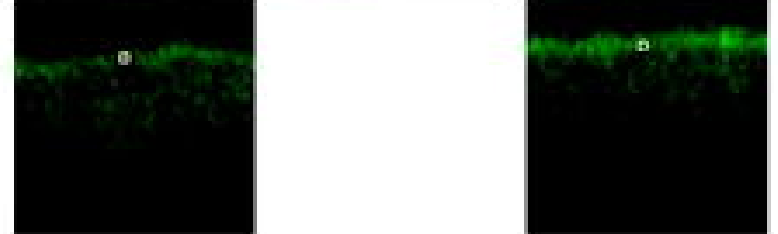

0.66
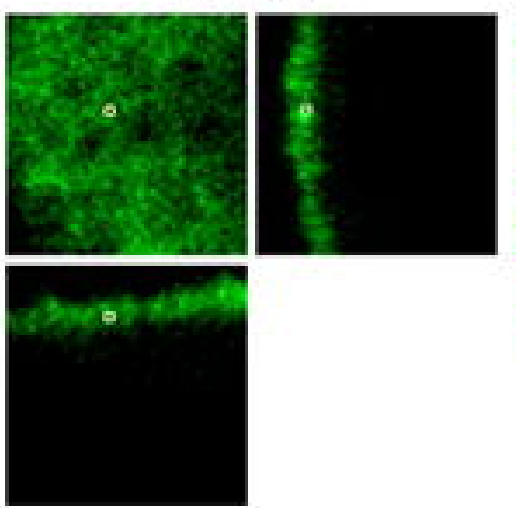

0.99

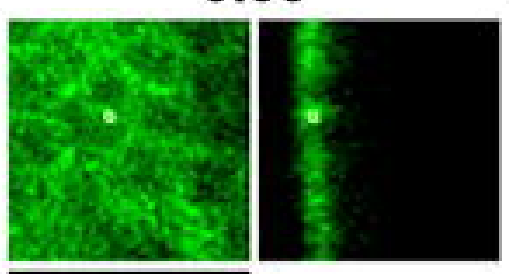

UVC $\left(\mathrm{J} / \mathrm{cm}^{2}\right)$ 

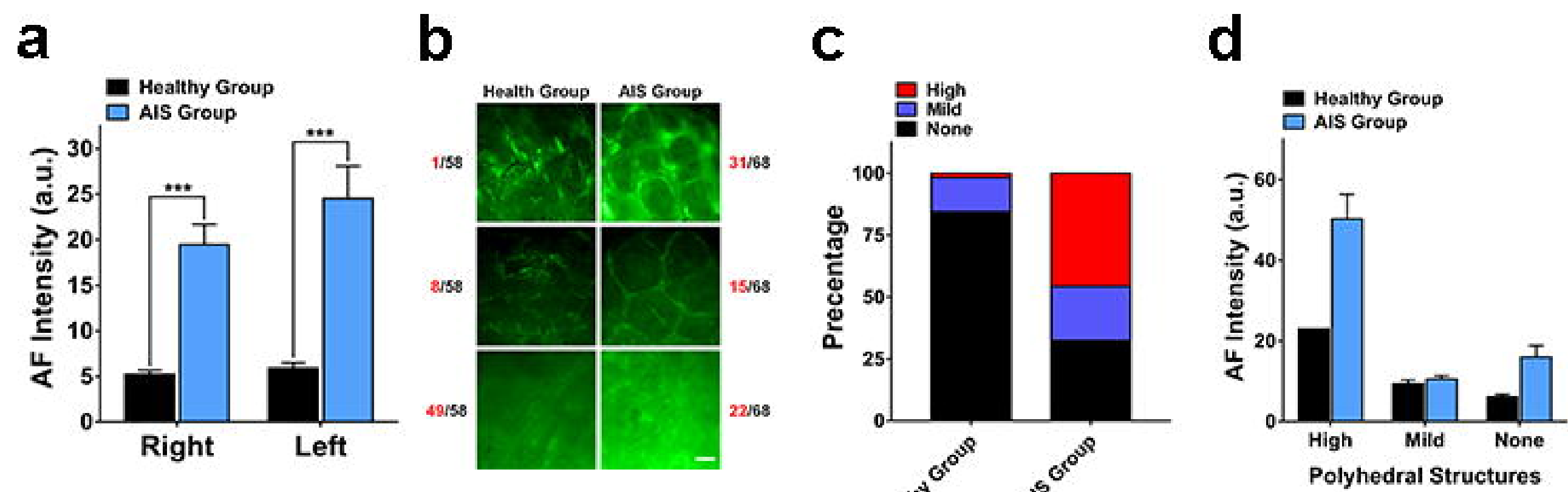

bioRxiv preprint doi: https://doi.org/10,1101/564112; this version posted May 30,2020. The copyright holdentor this preprint thichich was

Polyhedral Structures
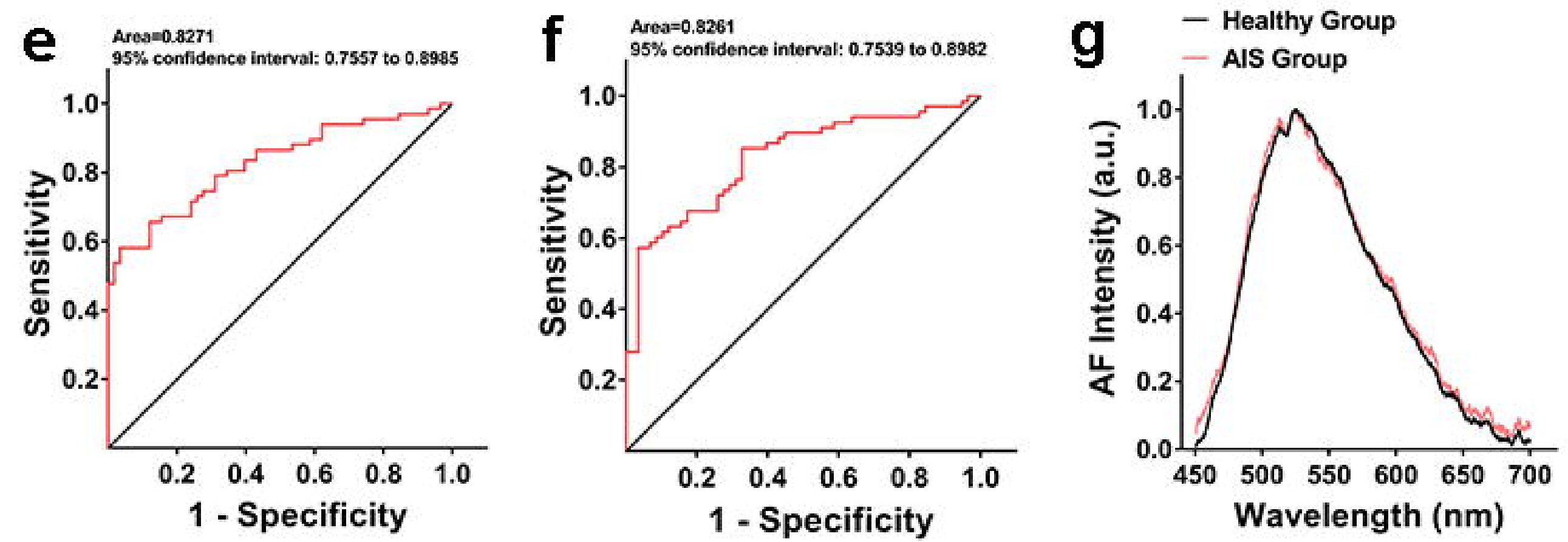

h
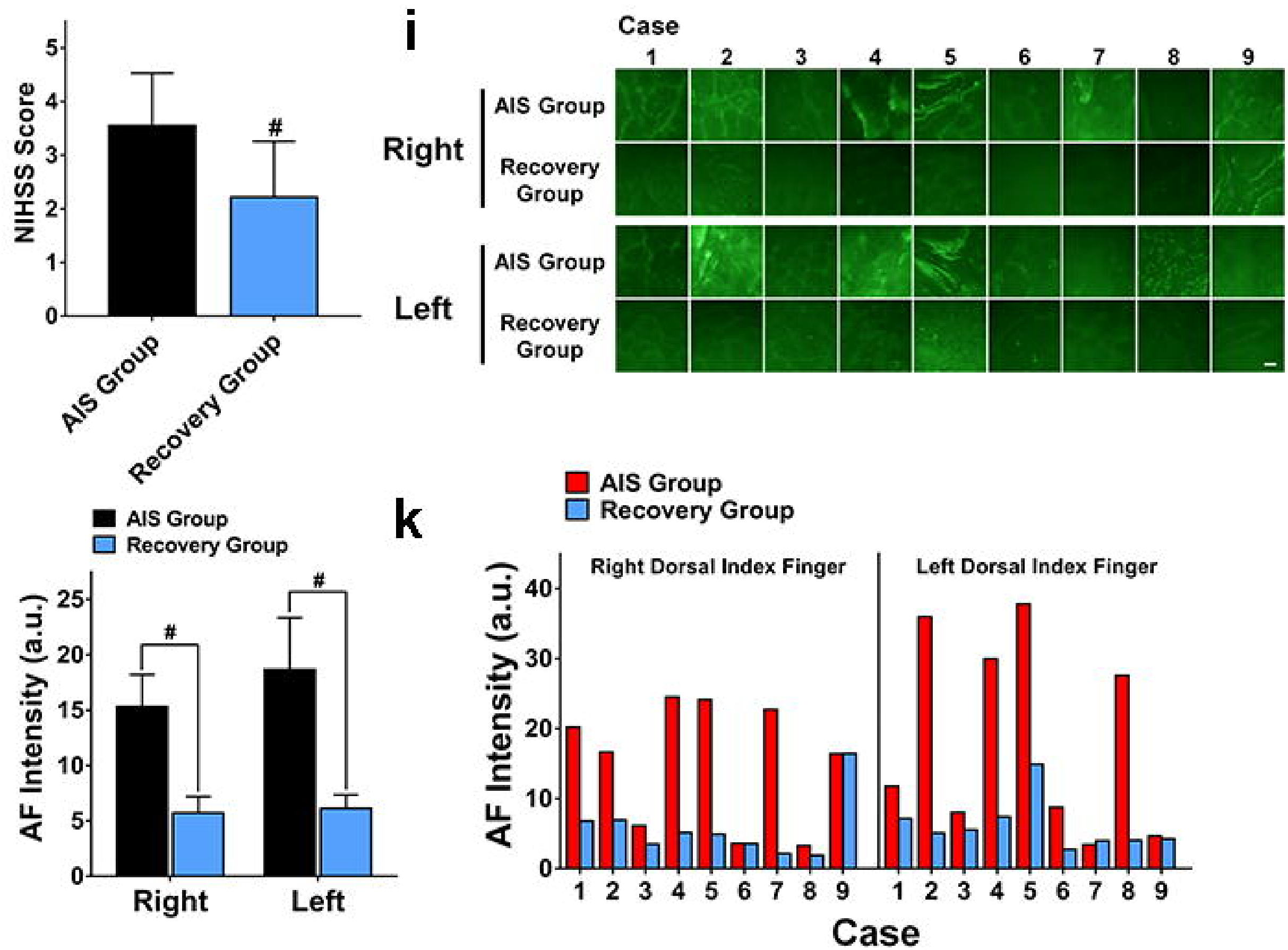
a $\quad$ b

d
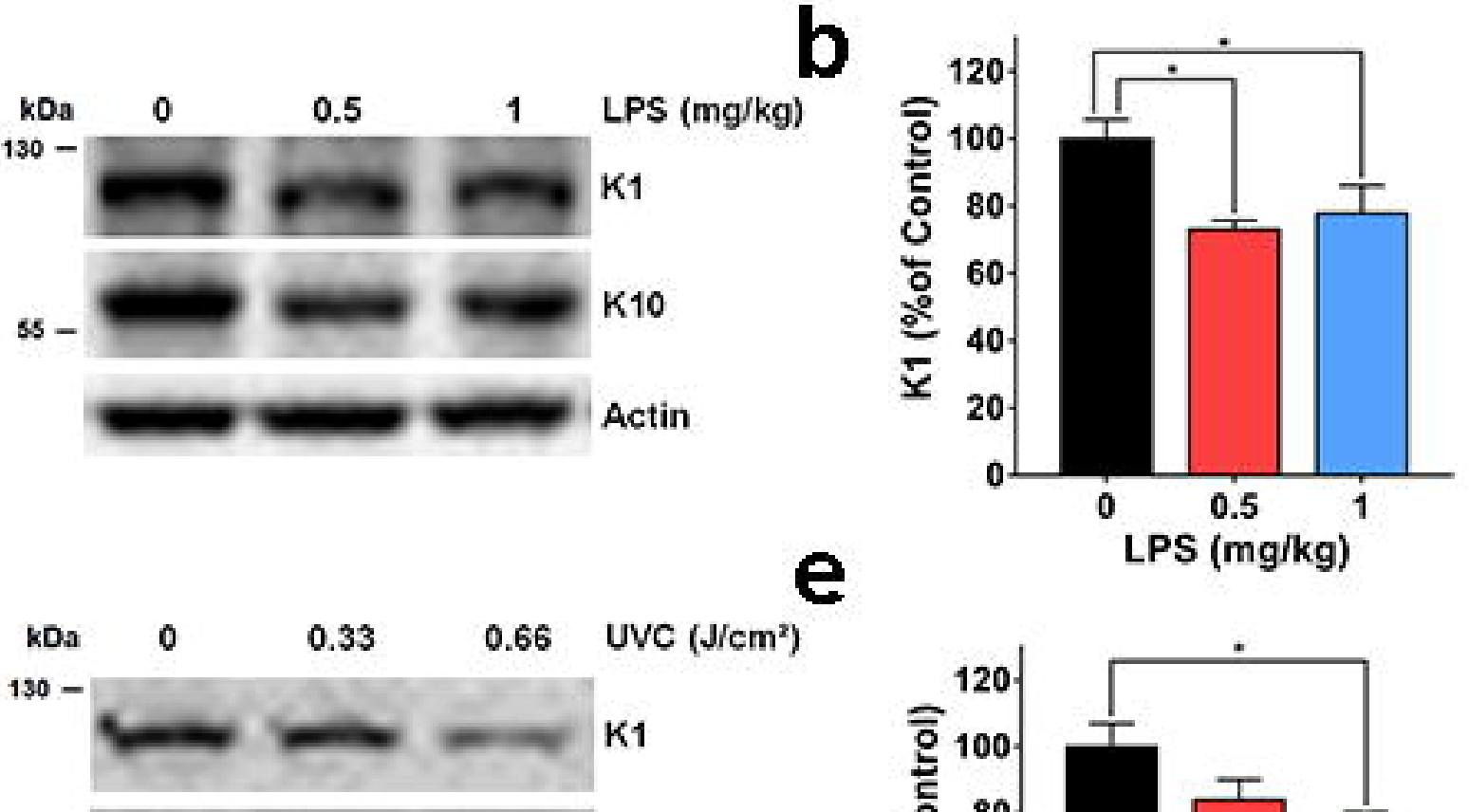
bioRxiv preprint doi: https://doi.org/10.1101/564112; this version posted M
not certified by peer

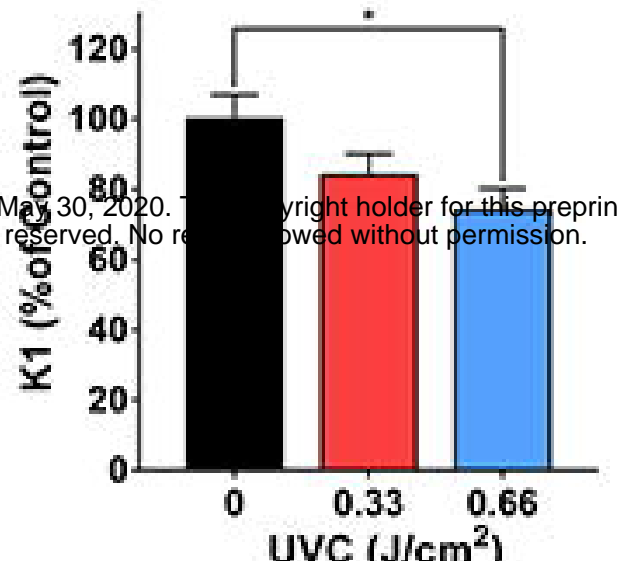

$9 \quad k D a \frac{\text { Vechicle }}{\text { con Uvc }} \frac{\text { NAC }}{\text { con UVC }}$

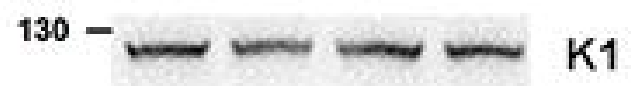

I

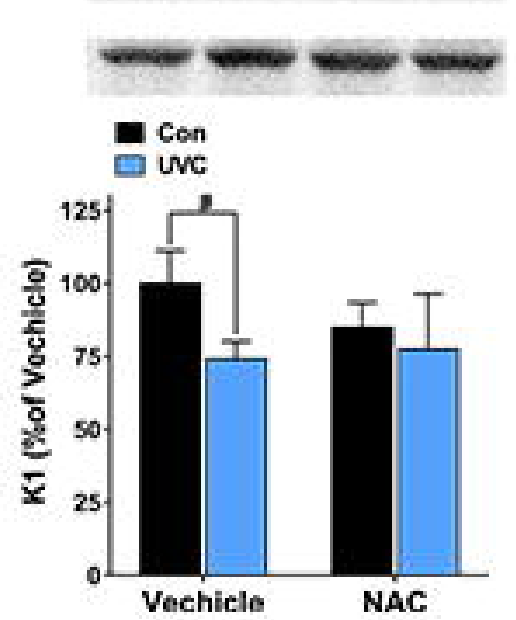

Actin

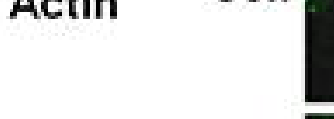

k

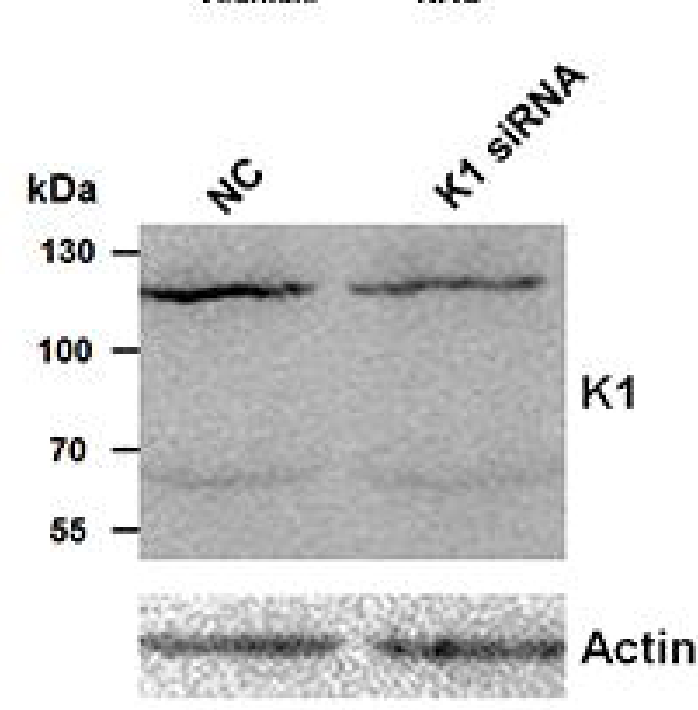

I

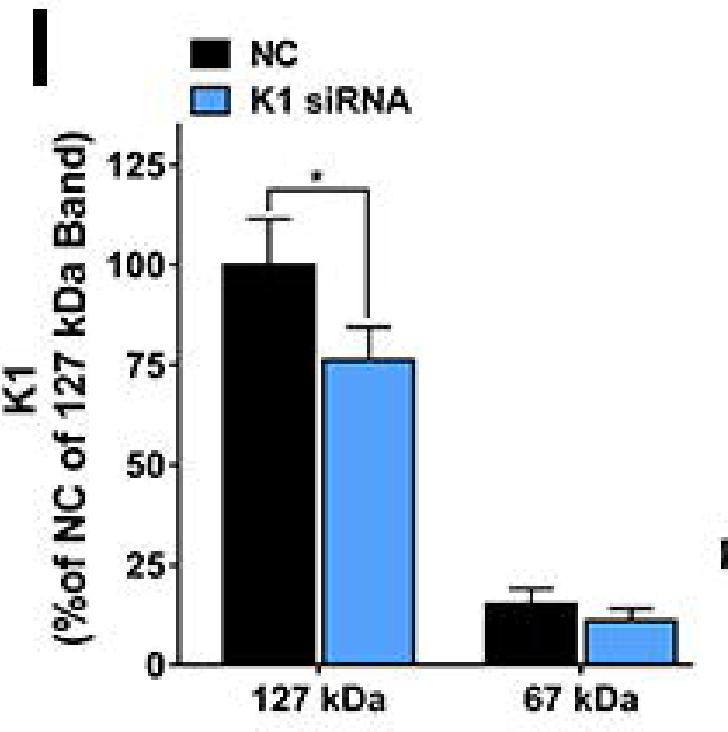

n

UVC

K1 siRNA
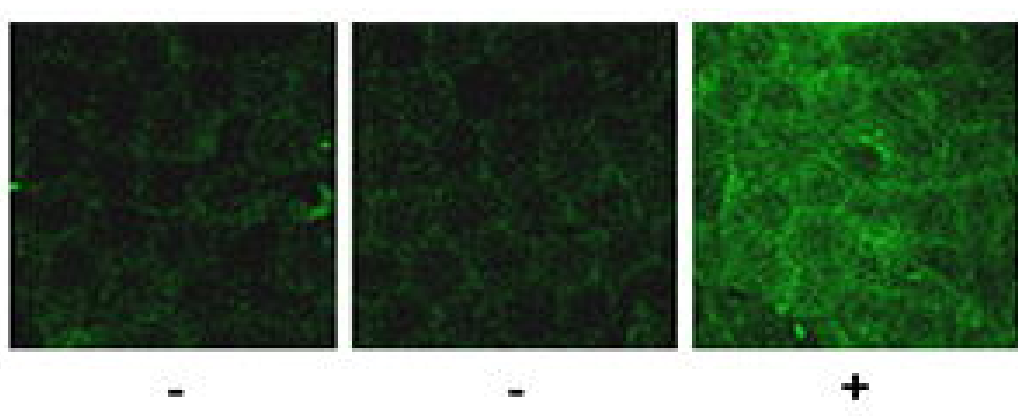
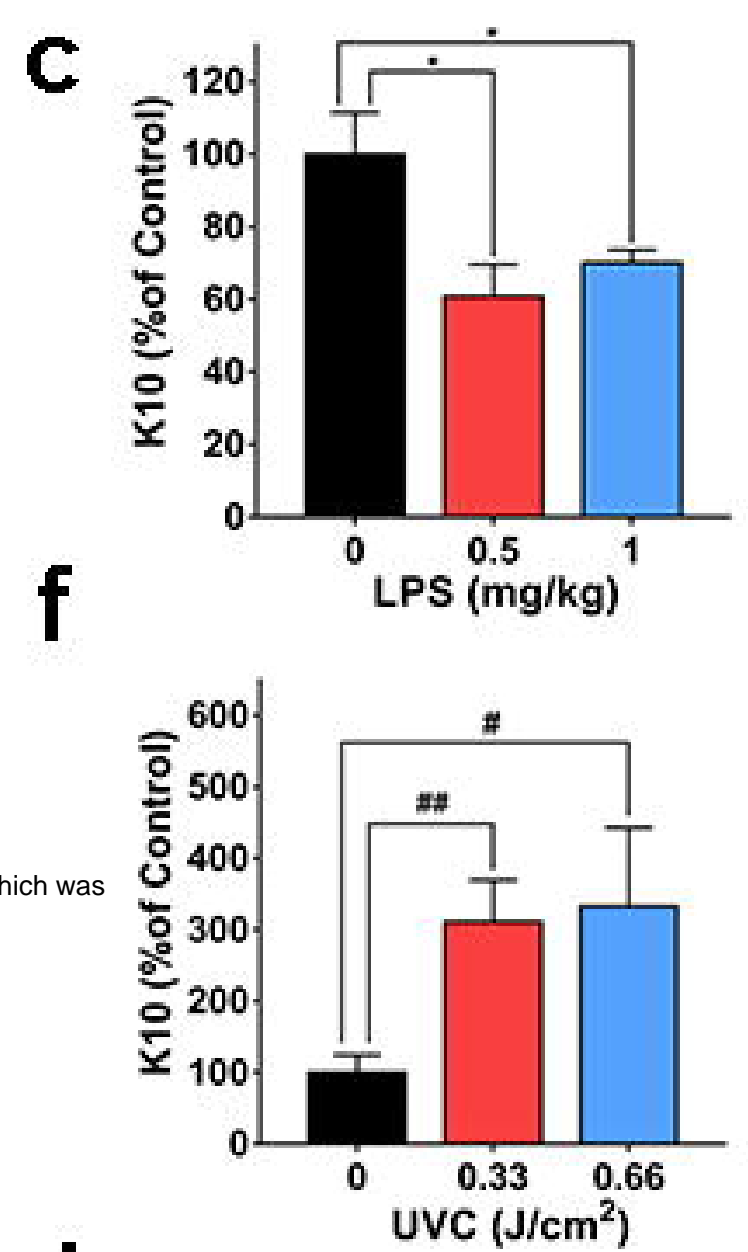

j

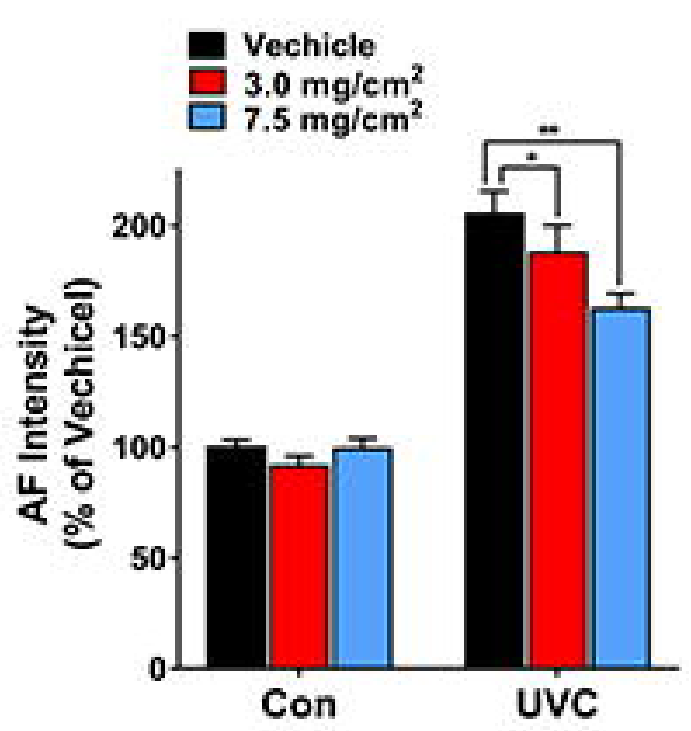

m
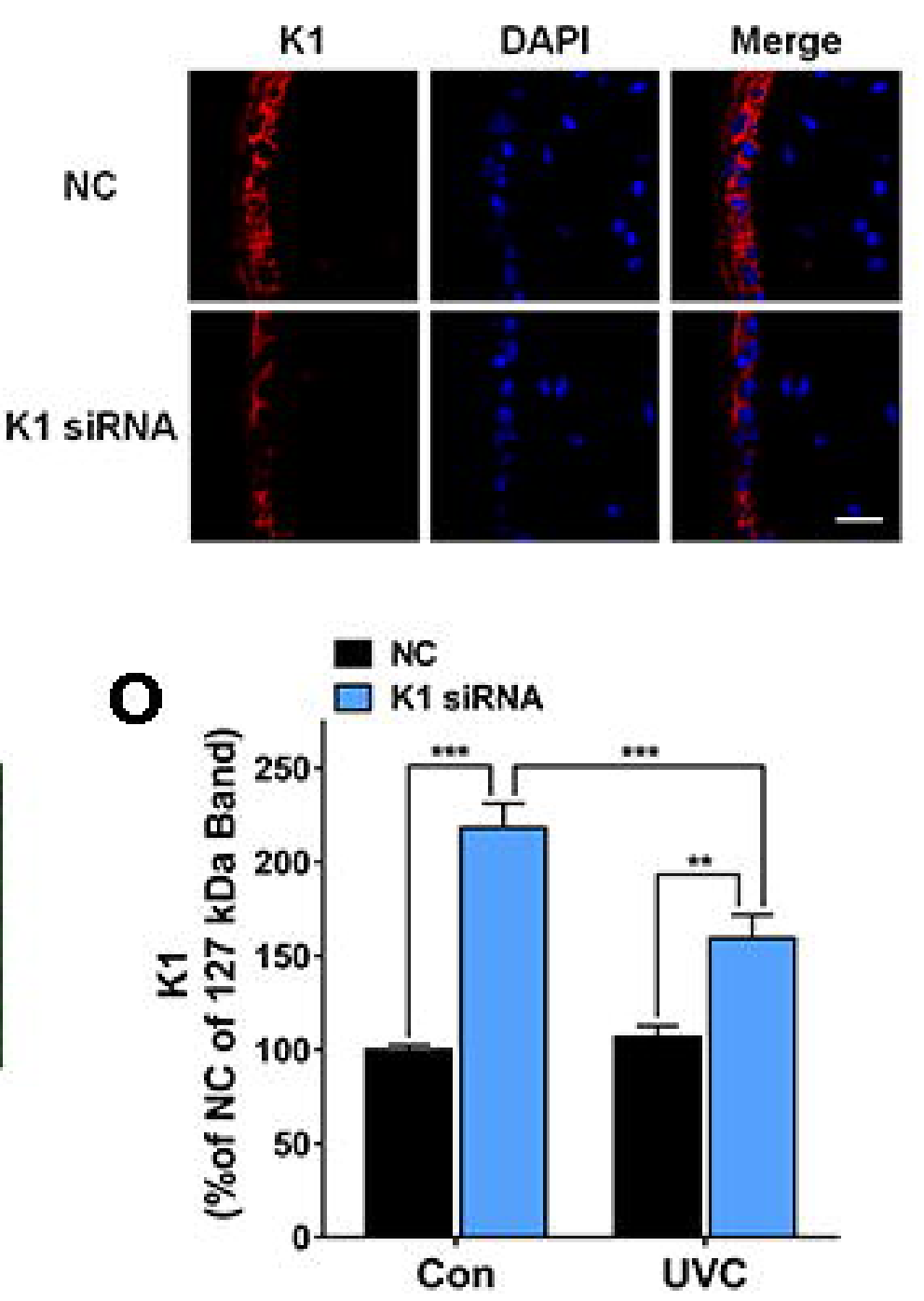
[LPS] vs. Log [Cytokine]

Cytokine

IL-1 $\beta$

IL-2.

IL-5.

IL-6.

IL-10.

IL-12(p40).

G-CSF.

MCP-1. $p$ value

0.0007

0.0006

0.0143

$<0.0001$

0.0029

0.0043

$<0.0001$

0.0040

R

0.7815

0.6169

0.8608

0.7122

0.6910

0.9250

0.6949
AF vs. Log [Cytokine]

$p$ value

0.0141

0.0153

0.0258

0.0427

0.0081

0.0106

0.0183

0.0342
$\mathbf{R}$

0.6181

0.6122

0.5723

0.5289

0.6549

0.6374

0.5990

0.5485 


\section{[LPS] vs. [Cytokine]}

Cytokine

MIP-1a MIP-1 $\beta$

RANTES. $p$ value

0.0032

0.0080

0.0003

R

0.7072

0.6555

0.8075
AF vs. [Cytokine]

$p$ value

0.0012

0.0031

0.0002

R

0.7542

0.7085

0.8184 